Ecological Applications, 1997, v.7, n.4, pp. 1311-1329.

Print ISSN: 1051-0761

Online ISSN: 1939-5582

DOI: 10.1890/1051-0761(1997)007[1311:EPSSBU]2.0.CO;2

http://www.esajournals.org/loi/ecap

http://www.esajournals.org/doi/pdf/10.1890/1051-

0761(1997)007\%5B1311\%3AEPSSBU\%5D2.0.CO\%3B2

(C) 1997 by the Ecological Society of America 


\title{
ENHANCING PERCID STOCKING SUCCESS BY UNDERSTANDING AGE-0 PISCIVORE-PREY INTERACTIONS IN RESERVOIRS
}

\author{
Nicholas S. Donovan, ${ }^{1,3}$ Roy A. Stein, ${ }^{1,4}$ And Matthew M. White ${ }^{2}$ \\ ${ }^{1}$ Aquatic Ecology Laboratory, Department of Zoology, The Ohio State University, 1314 Kinnear Road, \\ Columbus, Ohio 43212 USA \\ ${ }^{2}$ Department of Biological Sciences, Ohio University, Athens, Ohio 45701 USA
}

\begin{abstract}
Though young-of-year (YOY) saugeyes (Stizostedion vitreum $\times$ S. canadense) are routinely stocked in spring to create and maintain percid fisheries, their growth and survival to fall vary greatly among Ohio reservoirs, as well as among years within a reservoir. To understand the relative importance of size-dependent and size-independent mechanisms during ontogeny that underlie variable stocking success of saugeye, we quantified the role of stocking date and prey density (zooplankton and ichthyoplankton, i.e., larval gizzard shad [Dorosoma cepedianum]) in field enclosure, pond, and reservoir experiments. In $1-\mathrm{m}^{3}$ enclosures, ichthyoplankton density $(0,5,10$, or 20 larval gizzard shad/ $\mathrm{m}^{3}$ ) did not influence time to switch to piscivory by saugeye (all switched in $<12 \mathrm{~h}$ ); saugeye in enclosures with ichthyoplankton, regardless of density, grew faster than those without ichthyoplankton. In 0.4-ha ponds, saugeye growth and survival did not differ between ponds with zooplankton plus macrobenthic prey and ponds with those prey plus small ichthyoplankton $(<10 \mathrm{~mm})$.

In reservoir experiments, we evaluated how time in reservoirs, zooplankton density, and peak density (as well as date) of ichthyoplankton influenced saugeye growth and survival during 1991-1994 ( $N=31$ reservoir-years). In 1993, we attempted to bracket the ichthyoplankton peak in five Ohio reservoirs by stocking two genetically identifiable cohorts of saugeye $2 \mathrm{wk}$ apart in spring. For all reservoirs, those saugeye stocked before the ichthyoplankton peak grew larger than those stocked after the ichthyoplankton peak by 1 October. In 1994, we hypothesized that saugeye might overexploit local populations of ichthyoplankton when stocked at a single site. We paired 10 reservoirs $(N=5$ pairs $)$ with one reservoir of each pair scatter-stocked (i.e., saugeye numbers equally divided among five sites) and the second point stocked (i.e., at a single site). Stocking method did not influence saugeye survival; late gizzard shad spawning, coupled with low larval densities, yielded poor saugeye survival in 1994. However, YOY saugeye were considerably larger in fall 1994 than in fall 1993, when gizzard shad appeared earlier and produced more larvae. Zooplankton density at stocking influenced neither growth nor survival. Increased time in reservoirs increased fall size but did not influence survival. Although saugeye growth and survival during their first year were unrelated, both measures of stocking success critically depended on gizzard shad availability. Across all years, saugeyes stocked before ichthyoplankton peaks were large (as a result of their ability to consume fast-growing gizzard shad through summer), but survived poorly to fall (perhaps owing to early, high predatory mortality). Conversely, saugeyes stocked after ichthyoplankton peaks were small in fall (for they were unable to exploit large gizzard shad) but survived better (perhaps because gizzard shad provided a predatory buffer). By manipulating stock date relative to ichthyoplankton peaks, fisheries managers can either increase saugeye size or survival to fall, but not both.
\end{abstract}

Key words: fisheries management; gizzard shad; growth; ontogeny; Percidae; piscivory; predatorprey; recruitment; reservoir fisheries; sauger-walleye hybrid; saugeye; stocking; survival; young-ofyear fishes.

\section{INTRODUCTION}

Growth during early life stages often regulates recruitment into adult populations (Connell et al. 1984,

Manuscript received 24 July 1996; revised 10 February 1997; accepted 13 February 1997.

${ }^{3}$ Present address: Osteoporosis and Arthritis Research Group, University of California-San Francisco, San Francisco, California 94143-1250.

${ }^{4}$ Address reprint requests to this author.
Werner and Gilliam 1984). For highly fecund species, progeny are generally small, suffering high mortality from abiotic and biotic sources (May 1974, Houde 1987, Louda et al. 1990). Survival through ontogeny may depend on becoming sufficiently large to consume progressively larger prey, to avoid size-dependent predators, and to persist in the face of competitors (Paine 1976, Werner and Gilliam 1984, Condit et al. 1994, Ludsin and DeVries 1997). Juveniles of species with 
indeterminate growth (e.g., many plants and fishes) can exhibit high variability in growth among individuals within a year or among years (DeAngelis and Coutant 1992, Baylis et al. 1993, Rice et al. 1993, Lauenroth et al. 1994), causing variable recruitment into adult populations (Antonovics and Levin 1980, Adams and DeAngelis 1987, Madenjian and Carpenter 1991).

Within fishes, recruitment success often varies widely within a year (Crecco and Savoy 1985, Victor 1986, Houde 1994) and among years (Cushing 1975, Forney 1976, Doherty and Fowler 1994). For individuals within a cohort, probability of survival during the first growing season (typically summer in north temperate regions) depends both on size-independent stressors (e.g., egg mortality due to siltation; Kramer and Smith 1962) as well as size-dependent ones (e.g., successful competition for resources; Stein et al. 1972, Marschall and Crowder 1995, vulnerability to predation; Healey 1982, Post and Prankevicius 1987). Survival through the first winter may be the final critical period for young-of-year (YOY) fishes before recruiting to adult populations (Gutreuter and Anderson 1985, Shuter and Post 1990, Ludsin and DeVries 1997). Large fish enjoy higher survival overwinter (largemouth bass [Micropterus salmoides], Aggus and Elliott 1975, Adams et al. 1982, Ludsin and DeVries 1997; Stizostedion spp., Forney 1976, Post and Evans 1989; Esox spp., Carline et al. 1986; percids and smallmouth bass [M. dolomieui], Shuter and Post 1990), given their greater lipid reserves (Shuter and Post 1990, Thompson et al. 1991, Ludsin and DeVries 1997), their lower mass-specific metabolism compared to small fish (Oliver et al. 1979, Shuter and Post 1990), or their lower vulnerability to predation (Forney 1976). Thus, growth during the first summer and size entering winter likely regulates recruitment of temperate warmwater fishes.

For piscivores, availability of appropriate prey during ontogeny drives growth and survival (Werner and Gilliam 1984). Thus, hatching should be timed to take advantage of appropriately sized prey for larvae ( $\mathrm{Li}$ and Mathias 1982), such that larvae and juveniles can grow quickly beyond sizes vulnerable to starvation (Lasker 1975) and size-selective predation (Crowder 1980, Healey 1982, Post and Prankevicius 1987, Rice et al. 1987a). Differences in growth rate between successful and unsuccessful individuals need not be very large. In a modeling exercise, increasing growth rates of larval bloater (Coregonus hoyi) by $0.2 \mathrm{~mm} / \mathrm{d}$ increased larval survival by about $50 \times$ (Luecke et al. 1990). Conversion from zooplanktivorous or benthivorous feeding to piscivory greatly increases growth (largemouth bass, Aggus and Elliott 1975, Gutreuter and Anderson 1985, Olson 1996; J. E. Garvey, Aquatic Ecology Laboratory, The Ohio State University, unpublished data; Esox spp., Carline et al. 1986; Stizostedion spp., Forney 1976, B. L. Johnson et al. 1988, Buijse and Houthuijzen 1992), and can be regulated by prey type (Werner and Gilliam 1984), density (Mathias and $\mathrm{Li}$ 1982), and size (Jackson et al. 1992), or some combination of these three factors (Mittelbach 1981, Stahl and Stein 1994). Timing of this diet conversion can govern summer growth potential of piscivores (DeAngelis and Coutant 1982, Werner and Gilliam 1984, Gutreuter and Anderson 1985, Adams and DeAngelis 1987, Fox 1989, Madenjian and Carpenter 1991) and depends on spawning time of both predators and prey, and their subsequent growth rates (Olson 1996). Because piscivores are gape-limited predators, rapid growth of prey fishes during summer can reduce prey vulnerability to predation, reducing piscivore growth through fall (Hambright et al. 1991). Largemouth bass spawned early can consume fish prey earlier (Phillips et al. 1995) as well as later in summer than those spawned later (Adams and DeAngelis 1987, Phillips et al. 1995). Individual-based modeling exercises have successfully simulated length distributions at the end of summer based on early predator-prey length ratios (Adams and DeAngelis 1987, Madenjian and Carpenter 1991, Madenjian et al. 1991).

Despite increased production potential of saugeye (Stizostedion vitreum $\times$ S. canadense) in hatchery ponds (Culver et al. 1993, Qin et al. 1994), and the generally greater growth of saugeye than walleye (Stizostedion vitreum) in hatcheries (Malison et al. 1990, Siegwarth and Summerfelt 1990, Qin et al. 1994) and reservoirs (B. L. Johnson et al. 1988), survival and growth to fall of stocked fingerling saugeye vary greatly among reservoirs and across years within the same reservoir (Stahl and Stein 1994, Donovan et al. 1996). This variability limits the ability of fisheries managers to consistently create and maintain recreational saugeye fisheries.

Hence, to explore the relative importance of sizedependent and size-independent mechanisms during saugeye ontogeny, we conducted replicated small-scale and whole-reservoir experiments during 1991-1994. In small-scale experiments, we assessed how larval gizzard shad density influenced saugeye conversion to piscivory, as well as their growth following conversion, fully expecting that gizzard shad density would influence piscivory, and piscivory would increase saugeye growth. In reservoir experiments, we quantified how time of stocking, prey availability at the time of stocking, and saugeye density near the stock site influenced saugeye diet, growth, and survival through fall, and overwinter. Our expectation was that faster growth would translate to higher survival. However, in contrast to our expectations, first-year growth and survival of fingerling saugeye stocked in Ohio reservoirs were unrelated. Specifically, peak density of larval gizzard shad foreshadowed their abundance later in summer, thus providing a predatory buffer that positively influences saugeye survival via size-independent processes. Then, following a period of weeks after saugeye stocking in which small initial size differences were maintained, but not increased, large differences in saugeye 
size developed by fall due to size-dependent interactions with age- 0 gizzard shad. By understanding these interactions with age-0 gizzard shad as prey, we then suggest stocking strategies that enhance growth, survival, and return to the creel for saugeye.

\section{Materials And Methods}

\section{Enclosure experiment}

To determine how gizzard shad density influences conversion to piscivory and growth by saugeye, we conducted an enclosure experiment at Hebron State Fish Hatchery (Hebron, Ohio) during spring 1993. Specifically, we sought to extend the findings of Stahl and Stein (1994), who documented that time to piscivory did not differ between 20 and 100 larval gizzard shad/ $\mathrm{m}^{3}$. Hence, we tested four low-density treatments of 0 , 5, 10, or 20 larval gizzard $\mathrm{shad} / \mathrm{m}^{3}$. Experimental enclosures were $0.01-\mathrm{mm}$ clear plastic bags $(107 \times 76$ $\times 178 \mathrm{~cm})$ stapled onto wooden frames associated with a walkway constructed in a hatchery pond at Hebron State Fish Hatchery, Hebron, Ohio. This experiment was composed of two parts: (1) the "switch to piscivory" component quantified the time required for saugeye to become piscivorous in treatments with larval gizzard shad, and (2) the saugeye "growth" component quantified saugeye growth during $13 \mathrm{~d}$ immediately after conversion across the four treatments.

Switch to piscivory.-On 26 May 1993, 80 enclosures were filled with $\sim 1 \mathrm{~m}^{3}$ of water filtered through a $500-\mu \mathrm{m}$ mesh net. That night $0,5,10$, or 20 larval gizzard shad $(16.8 \pm 0.32 \mathrm{~mm}, N=30)$ were added to each of 20 enclosures. Enclosures were randomly selected to be dismantled at $12,24,48,96$, or $144 \mathrm{~h}$ ( $N=4$ replicates/treatment), to determine the time to conversion in each treatment. During 1000-1200 the next day, two saugeye $(35.4 \pm 0.21 \mathrm{~mm}, N=40 ; 0.29$ $\pm 0.004 \mathrm{~g}, N=36$ ) were added to each enclosure (hour 0 for reference). At hour 0 zooplankton were sampled by taking two $0.75-\mathrm{m}$ water columns with a clear Lexan tube sampler $(7.3 \mathrm{~cm}$ inside diameter, $1.6 \mathrm{~mm}$ thick; see DeVries and Stein 1991) from each enclosure, which were sieved through a 54- $\mu \mathrm{m}$ mesh net and preserved in $70 \%$ ethanol (EtOH). During dismantling, we pumped all but $\sim 10 \mathrm{~L}$ of water from the enclosure through a hose covered by a $500-\mu \mathrm{m}$ mesh net. Enclosure sides were rinsed with filtered water; remaining water was filtered by lifting the enclosure, cutting a hole in the bottom, and passing the water through a $500-\mu \mathrm{m}$ mesh net held below. Saugeye were preserved on wet ice; gizzard shad were preserved in $95 \% \mathrm{EtOH}$.

Growth.-Based on results of the "switch to piscivory" trials, treatments were adjusted to include replacement of consumed gizzard shad to quantify saugeye growth at each gizzard shad density. Larval gizzard shad were added on days $1,4,7$, and 10 to maintain densities of $0(N=8$ replicates $), 5(N=4), 10(N=$ $4)$, or 20 larval gizzard $\mathrm{shad} \cdot \mathrm{m}^{-3} \cdot$ saugeye $^{-1}(N=2)$ through $13 \mathrm{~d}$. Mean gizzard shad biomass $\cdot$ saugeye $\mathrm{e}^{-1} \cdot \mathrm{d}^{-1}$ during the experiment was $0.065,0.13$, and $0.26 \mathrm{~g}$ in the 5,10 , and $20 \mathrm{gizzard} \mathrm{shad} \cdot \mathrm{m}^{-3}$. saugeye $^{-1}$ treatments, respectively [gizzard shad biomass was estimated using the regression model ln(gizzard shad mass in grams $)=4.6762 \times \ln ($ gizzard shad total length in millimeters) -17.5839 ; gizzard shad size range 11$19.9 \mathrm{~mm}, r^{2}=0.9, P<0.0001 ;$ J. M. Dettmers, Illinois Natural History Survey, Alton, Illinois, unpublished data]. All enclosures were sampled for zooplankton (on days 7 and 13) and dismantled as in the "switch to piscivory" trials. Within-enclosure temperatures ranged from $17.5^{\circ}$ to $23^{\circ} \mathrm{C}$; dissolved oxygen surface to bottom ranged from 8.2 to $14.8 \mathrm{mg} / \mathrm{L}$ as measured by a YSI meter (Yellow Springs Instrument Company, Yellow Springs, Ohio).

For zooplankton analysis, cladocerans were identified to genus and copepods were identified as calanoids, cyclopoids, or nauplii. Rotifers were not counted. For abundant taxa, a minimum of 50 individuals per sample was counted from a thoroughly mixed subsample. For rare taxa, counting proceeded until either 50 individuals or the entire sample was counted, if the extrapolated number of individuals in each taxon was $>25$ individuals/sample after one-eighth of the sample was counted. If the calculated number was $<25$ individuals, the estimated abundance and density of that taxon were based on the number counted in the initial one-eighth of the sample. Numbers were converted to density estimates (number per liter).

\section{Pond experiment}

To determine how piscivory and its positive effects on growth and survival were influenced by stocking small saugeye before larval gizzard shad hatch, we completed a pond experiment at Hebron State Fish Hatchery in spring 1994. All ponds were 0.4 ha, 0.5$1.5 \mathrm{~m}$ deep, and without vegetation. Our experiment consisted of two treatments: (1) two replicate ponds of saugeye $(21.6 \pm 0.1 \mathrm{~mm}, 0.074 \pm 0.001 \mathrm{~g}, N=25)$ with zooplankton and macrobenthic prey only and (2) three replicate ponds of saugeye with these prey and larval gizzard shad. In addition, one reference pond contained larval gizzard shad, but no saugeye.

Ponds were filled on 29 April 1994. Fifty ripe adult gizzard shad $(270.4 \pm 6.5 \mathrm{~mm}, N=10)$, collected from Buckeye Lake (Licking-Fairfield Counties, Ohio), were added randomly to ponds designated as larval gizzard shad treatment ponds on 3 May. Saugeye $(N=992 \pm$ 19 per pond) were stocked into ponds on 6 May, before gizzard shad hatched. We estimated the number of saugeye added to each pond by counting the number of saugeye in a 109-g sample $(N=3)$. Beginning 9 May, larval gizzard shad were sampled daily ( $N=2$ tows) by hand-hauling a $0.5 \mathrm{~m}$ diameter, $500-\mu \mathrm{m}$ mesh net at the surface for about half the pond length; a mouthmounted flow meter provided estimates of water volume filtered. Samples were preserved in $95 \% \mathrm{EtOH}$. 
Beginning on the day of saugeye stocking, zooplankton were sampled with a clear Lexan tube sampler (see previous description in Enclosure experiment: switch to piscivory) at the deep end of each pond every 3rd d. Replicates were taken from each pond by sieving two 1-m tube hauls per sample through a 54- $\mu \mathrm{m}$ mesh net, and preserved in $70 \% \mathrm{EtOH}$. On days of zooplankton sampling, 10-20 saugeye per pond were collected for diet analysis during the $2 \mathrm{~h}$ before dark with a 4.6 $\times 1.8 \mathrm{~m}$ seine (3.2-mm mesh). Mean daily water temperatures ranged from $13.6^{\circ}$ to $24.7^{\circ} \mathrm{C}$.

All ponds were drained on 2 June 1994. Zooplankton densities were determined as described for the enclosure experiment, except we also measured (nearest $0.001 \mathrm{~mm}$ ) up to 22 individuals of each taxon from the anterior margin of the head to the base of either the tail spine of cladocerans or the caudal rami of copepods using a digitizing board. A minimum of 200 larval gizzard shad/sample were counted from a thoroughly mixed subsample of known volume. All saugeye were counted and at least 100 were measured (nearest millimeter) and weighed without stomach contents (nearest $0.001 \mathrm{~g}$ ) from each pond. Diets from up to $10 \mathrm{saug}$ eye.pond ${ }^{-1} \cdot$ date $^{-1}$ were identified, counted, and measured as per our zooplankton samples. Prey length was converted individually to mass from length-dry mass regressions (zooplankton from Dumont et al. 1975; macrobenthic organisms from G. G. Mittelbach, Kellogg Biological Station, Michigan State University, Hickory Corners, Michigan, unpublished data) to estimate biomass per saugeye (grams per gram). Dry mass of saugeye was estimated from the following independent regression equation: dry mass $=-0.018+0.21$ wet mass ( $r^{2}=0.97$, length range $25-91 \mathrm{~mm}, N=319$; Stahl and Stein 1994).

\section{3 reservoir experiment}

We designed the 1993 reservoir experiment to quantify how stocking date and prey availability influenced saugeye growth and survival through fall. We stocked saugeye into five, medium-sized (344-1145 ha) Ohio reservoirs (Table 1), representing an historical range of saugeye growth and survival (M. R. Austin, Ohio Division of Wildlife, Xenia, Ohio, personal communication). Mean secchi transparencies were $<2 \mathrm{~m}$, few aquatic macrophytes occurred, and gizzard shad dominated the piscine forage base in all reservoirs.

Saugeye were stocked at a density of 250 saugeye/ha twice, 2 wk apart (stock 1, May 18 or 19; stock 2, June 2 , 3, or 4; Table 1), with genetically identifiable cohorts. Stocking dates were selected to maximize differences in larval gizzard shad abundance, stock 1 at high prey and stock 2 at low prey. Due to fewer saugeye available for stock 2, Caesar Creek, Piedmont, and Tappan reservoirs were stocked with 168, 0, and 153 saugeye/ha, respectively (Table 1 ).

Saugeye rearing and protein determination.-Male sauger were collected from the Ohio River and held at
Seneccaville Hatchery before being transported to Seneca Lake (Guernsey and Noble Counties) and Salt Fork Lake (Guernsey County) to fertilize walleye eggs stripped from ripe females captured in trapnets. Fertilization occurred during 25 March-9 April. White muscle tissue was sampled with a biopsy needle from the adult sauger-walleye pair that provided each batch of fertilized eggs. Muscle samples were transported on ice to the laboratory. Fertilized eggs of each cross were transported to Senecaville Fish Hatchery, where they were held separately until progeny type was determined.

The locus used to genetically mark the saugeye cohorts was Muscle Protein (MP, muscle myogen; see Uthe and Ryder 1970). This locus is polymorphic for two common alleles (designated 100 and 70 herein) within North American walleye populations (White and Schell 1995). Conversely, sauger are monomorphic for the MP 100 allele (Todd 1990, White and Schell 1995). When crossed with sauger males, walleye females homozygous for the 100 allele produce only MP 100/100 homozygous saugeye offspring, whereas walleye females homozygous for the MP 70 allele produce only MP 100/70 heterozygous saugeye offspring. Because offspring from walleye heterozygous at MP could be homozygous or heterozygous, they were not used.

Tissues were homogenized in an equal volume of an aqueous buffer $(0.1 \mathrm{~mol} / \mathrm{L}$ Tris, $0.1 \mathrm{mmol} / \mathrm{L}$ EDTA, $0.01 \mathrm{mmol} / \mathrm{L}$ NAD [nicotinamide adenine dinucleotide], $0.01 \mathrm{mM}$ NADP [nicotinamide adenine dinucleotide phosphate], $\mathrm{pH} 7.0$ ), then centrifuged at 11000 $g$ at $4^{\circ} \mathrm{C}$ for $20 \mathrm{~min}$. Muscle protein was resolved on $12 \%$ starch gels (Starch Art, Smithville, Texas) in a Tris-borate-EDTA pH 8.6 buffer (Turner 1983), using standard electrophoresis techniques (Morizot and Schmidt 1990). Upon genotype determination, cohorts were identified and either raised for stocking our experiment or used in production.

To accelerate growth of groups to be stocked in May, eggs fertilized during 25-31 March were transferred to Hebron State Fish Hatchery on 31 March, where water temperatures have been historically greater than at Senecaville State Fish Hatchery. Eggs fertilized during 19 April were transferred to Hebron as fry on 22, 26, and 27 April. After hatching, 160000 saugeye each were released into 250.4 -ha ponds (a density recommended by Culver et al. 1993), taking care not to mix protein types. Stocks of each protein type were randomly assigned to reservoirs for the first stocking; reservoirs received the alternate protein type for the second stock. Saugeye were transported in aerated solutions $(0.5 \%$ salt $)$ of pond water and stocked at one point in each reservoir. Transportation to all reservoirs was $<2 \mathrm{~h}$; transportation mortality of saugeye is generally $<20 \%$ to Ohio reservoirs (Stahl and Stein 1994).

Prey availability. - Larval fish and zooplankton were sampled twice a week in each reservoir during the 2 wk before the first stocking to 2 wk after the second 
stocking, and then once a week through 30 June. Larval fish were collected from one site near and another (or two in Pleasant Hill) far from the stock site. Fish larvae were collected during the day by towing a $0.75-\mathrm{m} \mathrm{di}$ ameter, $500 \mu \mathrm{m}$, metered ichthyoplankton net along the surface at $1.5 \mathrm{~m} / \mathrm{s}$ for $2-5 \mathrm{~min}$, then preserved in $95 \%$ $\mathrm{EtOH}$, and counted as described for the enclosure experiment. Zooplankton were collected from two sites, near larval fish sampling sites, in each reservoir (four in Pleasant Hill). Zooplankton were collected during the day with a $0.31-\mathrm{m}$ diameter, 540- $\mu \mathrm{m}$ mesh net hauled vertically through the entire water column. Zooplankton were preserved in $70 \% \mathrm{EtOH}$ and counted as described previously.

Saugeye growth and survival.-Saugeye were collected twice a week from the first stocking to 2 wk after the second stocking, and then once a week through 30 June. Saugeye were collected with seines $(4.6 \times$ $1.8 \mathrm{~mm}$ ) from two sites (three 10-20 m transects/site), one near the stock site and one in likely saugeye habitat far from the stock site, in each reservoir. Either 10 saugeye were collected from each site, or seining continued until three successive hauls at a transect yielded no fish. Captured saugeye were placed on wet ice for diet and muscle protein analysis.

To determine survival of age- 0 saugeye in Pleasant Hill Reservoir through 2.5 mo after stocking, the entire shoreline was electrofished twice, on 5 and 11 August to generate a mark-recapture Peterson population estimate. To determine survival from stocking to fall, population estimates were performed during the first week of October in Caeasar Creek Lake, and Delaware and Pleasant Hill reservoirs. In Piedmont and Tappan reservoirs, an equal distance ( $>33 \%$ of the total) along the shoreline of each known substrate type was randomly sampled and population size was estimated using a regression model, number per hectare $=0.9763$ $\left(\log _{10}\right.$ [catch per hour] $)-0.424\left(r^{2}=0.74, P<0.0001\right.$, $N=30$; M. R. Austin, Ohio Division of Wildlife, Xenia, Ohio, unpublished data). To determine whether the size of YOY saugeye in the fall influenced their ability to overwinter, we estimated electrofishing catch per unit effort (CPUE) on 14 April 1994 in Pleasant Hill Reservoir and 29 March and 16 April 1994 in Delaware Reservoir. All saugeye captured during CPUEs and population estimates were measured (nearest millimeter, total length [TL]) and 10-20 saugeye per centimeter of length class were weighed (nearest $0.1 \mathrm{~g}$ ) and placed on wet ice for muscle protein and diet analysis.

To assign stock date to saugeye recaptured in seines, we identified protein type for all saugeye for which we analyzed diet contents (up to 26 saugeye.date ${ }^{-1}$. reservoir ${ }^{-1}$ ). Protein type also was determined for all saugeye recaptured via electrofishing in summer 1993, fall 1993, and spring 1994, for which we analyzed diet contents (generally 10 saugeye/10-cm size class; 20 saugeye/10-cm size class for abundant size classes). To estimate overall mean length of saugeye from each stock in summer and fall 1993 and spring 1994, saugeye unidentified as to protein type were assigned to a stock date by randomly selecting individuals from a $1-\mathrm{cm}$ class according to stock 1 :stock 2 protein ratios determined for each 1-cm class. In fall 1993, the only season when not all saugeye collected were weighed, we estimated individual mass by applying length-mass regressions developed for each reservoir (Donovan et al. 1996). Survival was estimated for each stock by dividing the population estimate of each stock, determined from length-class specific protein ratios, by the number of saugeye of each protein type stocked into a reservoir.

\section{4 reservoir experiment}

To determine how larval gizzard shad density at the saugeye stocking site influences saugeye success through fall, we quantified diet, growth, and survival of saugeye stocked via two methods into 10 reservoirs. Saugeye were stocked at a density of 250 saugeye/ha in six reservoirs on 18 May 1994. These reservoirs were those stocked in 1993 plus Charles Mill Lake (Richland and Ashland Counties). In addition, four reservoirs were stocked at a density of 600-782 saugeye/ha on 25 May 1994 (Table 1). Reservoirs were paired according to historical saugeye survival and larval gizzard shad production (Stahl et al. 1993; M. R. Austin, Ohio Division of Wildlife, Xenia, Ohio, personal communication). To increase larval gizzard shad availability per stocked saugeye, which might occur with high dispersal, one reservoir of each pair was "scatter" stocked, i.e., in reservoirs to receive 250 saugeye/ha, 50 saugeye/ha were stocked at five sites. The other set was "point" stocked, i.e., the historical method of stocking all 250 saugeye/ha at one location (Table 1).

Prey availability and saugeye growth and survival.- - Reservoirs stocked at a density of 600-782 saugeye/ha were sampled by electrofishing in the fall (29 September-12 October) to estimate mass in fall and oversummer survival. We did not sample for prey around stocking time in these reservoirs. In reservoirs stocked at a density of 250 saugeye/ha, zooplankton, larval fish, and age-0 saugeye were sampled as described for the 1993 reservoir experiment, with the following exceptions. Zooplankton, larval fish, and age-0 saugeye (when appropriate) were sampled twice weekly for $2 \mathrm{wk}$ before stocking to $4 \mathrm{wk}$ after stocking. In scatter-stocked reservoirs, seine sites (2-3 transects/ site) were established to collect saugeye near 2-4 stocking sites. Length distributions of age-0 gizzard shad were determined by collecting at least 50 individuals from a minimum of three sites via electrofishing on the dates of fall saugeye population estimates (26 September-4 October).

Analysis of diets.-Diet analysis of age-0 saugeye collected from reservoirs in 1993 and 1994 was as described for the pond experiment, except for fish prey. 
TABLE 1. Summary information from Ohio reservoirs stocked with saugeye during 1991-1994, including number, mean total length $(N=30$ saugeye/lake $)$, and mean wet mass $(N=30$ saugeye/lake $)$ at stocking as well as these characteristics on 1 October. Standard errors are given in parentheses. Identification numbers (ID no.) identify reservoirs in subsequent figures; first digits of these numbers represent the year of sampling (e.g., 10 and 34 were sampled in 1991 and 1993 , respectively). P denotes point-stocked reservoirs; S denotes scatter-stocked reservoirs.

\begin{tabular}{|c|c|c|c|c|c|c|c|c|}
\hline \multirow[b]{2}{*}{$\begin{array}{l}\text { Year } \\
\text { ID no. }\end{array}$} & \multirow[b]{2}{*}{ Reservoir (ha) } & \multirow{2}{*}{$\begin{array}{c}\text { No. } \\
\text { stocked/ } \\
\text { ha } \\
\text { (method) }\end{array}$} & \multirow[b]{2}{*}{ Stock date } & \multicolumn{4}{|c|}{ Mean sizes (1 SE) } & \multirow[b]{2}{*}{$\begin{array}{l}\text { Fall population } \\
\text { estimate } \\
(95 \% \mathrm{CI})\end{array}$} \\
\hline & & & & $\begin{array}{l}\text { Stock } \\
\text { length } \\
(\mathrm{mm})\end{array}$ & $\begin{array}{l}\text { Stock } \\
\text { mass } \\
(\mathrm{g})\end{array}$ & $\begin{array}{c}1 \text { Oct } \\
\text { length }(\mathrm{mm})\end{array}$ & $\begin{array}{l}1 \text { Oct mass } \\
(\mathrm{g})\end{array}$ & \\
\hline \multicolumn{9}{|l|}{1991} \\
\hline 10 & $\begin{array}{l}\text { Caesar Creek } \\
(1146)\end{array}$ & $\begin{array}{l}250 \\
(\mathrm{P})\end{array}$ & 21 May & $\begin{array}{l}33.7 \\
(0.43)\end{array}$ & $\begin{array}{c}0.214 \\
(0.009)\end{array}$ & $\begin{array}{c}174.9 \\
(1.05)\end{array}$ & $\begin{array}{l}43.7 \\
(0.83)\end{array}$ & $\begin{array}{c}10720 \\
(9000-12769)\end{array}$ \\
\hline 11 & $\begin{array}{l}\text { Delaware } \\
(526)\end{array}$ & $\begin{array}{l}250 \\
(\mathrm{P})\end{array}$ & 4 June & $\begin{array}{l}39.4 \\
(0.63)\end{array}$ & $\begin{array}{c}0.342 \\
(0.017)\end{array}$ & $\begin{array}{c}191.3 \\
(0.87)\end{array}$ & $\begin{array}{l}62.5 \\
(0.91)\end{array}$ & $\begin{array}{c}14350 \\
(12326-16710)\end{array}$ \\
\hline 12 & $\begin{array}{l}\text { Logan } \\
(162)\end{array}$ & $\begin{array}{l}212 \\
(\mathrm{P})\end{array}$ & 29 May & $\begin{array}{l}38.5 \\
(0.9)\end{array}$ & $\begin{array}{c}0.320 \\
(0.028)\end{array}$ & $\begin{array}{c}171.2 \\
(3.09)\end{array}$ & $\begin{array}{l}39.1 \\
(2.13)\end{array}$ & $\begin{array}{c}270 \\
(160-444)\end{array}$ \\
\hline 13 & $\begin{array}{l}\text { Pleasant Hill } \\
\text { (344) }\end{array}$ & $\begin{array}{l}250 \\
(\mathrm{P})\end{array}$ & 14 May & $\begin{array}{l}33.5 \\
(0.37)\end{array}$ & $\begin{array}{l}0.265 \\
(0.008)\end{array}$ & $\begin{array}{c}209.3 \\
(1.31)\end{array}$ & $\begin{array}{l}78.5 \\
(1.39)\end{array}$ & $\begin{array}{c}9197 \\
(7532-11230)\end{array}$ \\
\hline \multicolumn{9}{|l|}{1992} \\
\hline 20 & $\begin{array}{l}\text { Caesar Creek } \\
(1145)\end{array}$ & $\begin{array}{l}250 \\
(\mathrm{P})\end{array}$ & 19 May & 30.6 & 0.194 & $\begin{array}{r}213.5 \\
(0.89)\end{array}$ & $\begin{array}{l}76.5 \\
(1.03)\end{array}$ & $\begin{array}{c}6204 \\
(4933-7797)\end{array}$ \\
\hline 21 & $\begin{array}{l}\text { Charles Mill } \\
(547)\end{array}$ & $\begin{array}{l}250 \\
(\mathrm{P})\end{array}$ & 20 May & 31.8 & 0.220 & $\begin{array}{c}189.3 \\
(1.60)\end{array}$ & $\begin{array}{l}53.7 \\
(1.45)\end{array}$ & $\begin{array}{c}5590 \dagger \\
(1375-9783)\end{array}$ \\
\hline 22 & $\begin{array}{l}\text { Delaware } \\
(526)\end{array}$ & $\begin{array}{l}250 \\
(\mathrm{P})\end{array}$ & 3 June & 34.5 & 0.246 & $\begin{array}{c}211.1 \\
(0.89)\end{array}$ & $\begin{array}{l}76.5 \\
(1.03)\end{array}$ & $\begin{array}{c}5645 \\
(3679-8582)\end{array}$ \\
\hline 23 & $\begin{array}{l}\text { O'Shaughnessy } \\
\text { (336) }\end{array}$ & $\begin{array}{l}245 \\
(\mathrm{P})\end{array}$ & 28 May & 40.2 & 0.402 & $\begin{array}{c}187.2 \\
(2.20)\end{array}$ & $\begin{array}{l}51.2 \\
(2.14)\end{array}$ & $\begin{array}{c}845 \dagger \\
(317-1373)\end{array}$ \\
\hline 24 & $\begin{array}{l}\text { Paint Creek } \\
(482)\end{array}$ & $\begin{array}{l}250 \\
(\mathrm{P})\end{array}$ & 28 May & 34.3 & 0.257 & $\begin{array}{c}191.9 \\
(1.29)\end{array}$ & $\begin{array}{l}53.5 \\
(1.24)\end{array}$ & $\begin{array}{c}7353 \dagger \\
(5367-9412)\end{array}$ \\
\hline 25 & $\begin{array}{l}\text { Piedmont } \\
(935)\end{array}$ & $\begin{array}{l}250 \\
(\mathrm{P})\end{array}$ & 2 June & 41.3 & 0.416 & $\begin{array}{c}188.8 \\
(1.97)\end{array}$ & $\begin{array}{l}53.7 \\
(1.90)\end{array}$ & $\begin{array}{c}2649 \dagger \\
(1325-4132)\end{array}$ \\
\hline 26 & $\begin{array}{l}\text { Pleasant Hill } \\
\text { (344) }\end{array}$ & $\begin{array}{l}250 \\
(\mathrm{P})\end{array}$ & 12 May & 25.0 & 0.103 & $\begin{array}{c}233.5 \\
(1.27)\end{array}$ & $\begin{array}{c}105.4 \\
(2.00)\end{array}$ & $\begin{array}{c}2866 \\
(2004-4082)\end{array}$ \\
\hline 27 & $\begin{array}{l}\text { Tappan } \\
(951)\end{array}$ & $\begin{array}{l}250 \\
(\mathrm{P})\end{array}$ & 20 May & 35.7 & 0.283 & $\begin{array}{l}210.7 \\
(2.26)\end{array}$ & $\begin{array}{l}69.3 \\
(2.37)\end{array}$ & $\begin{array}{c}2395 \dagger \\
(1198-3449)\end{array}$ \\
\hline \multicolumn{9}{|l|}{1993} \\
\hline 30 & $\begin{array}{l}\text { Caesar Creek } \\
(1146)\end{array}$ & $\begin{array}{l}250 \\
(\mathrm{P})\end{array}$ & 18 May & $\begin{array}{l}30.4 \\
(0.22)\end{array}$ & $\begin{array}{c}0.193 \\
(0.009)\end{array}$ & $\begin{array}{l}202.4 \\
(0.98)\end{array}$ & $\begin{array}{l}66.5 \\
(1.05)\end{array}$ & $\begin{array}{c}6664 \\
(5464-8196)\end{array}$ \\
\hline 31 & Caesar Creek & $\begin{array}{l}165 \\
(\mathrm{P})\end{array}$ & 3 June & $\begin{array}{l}35.1 \\
(0.30)\end{array}$ & $\begin{array}{c}0.252 \\
(0.011)\end{array}$ & $\begin{array}{c}182.9 \\
(1.01)\end{array}$ & $\begin{array}{l}49.1 \\
(0.82)\end{array}$ & $\begin{array}{c}6661 \\
(5462-8193)\end{array}$ \\
\hline 32 & $\begin{array}{l}\text { Delaware } \\
(526)\end{array}$ & $\begin{array}{l}250 \\
(\mathrm{P})\end{array}$ & 19 May & $\begin{array}{l}30.8 \\
(0.29)\end{array}$ & $\begin{array}{c}0.225 \\
(0.006)\end{array}$ & $\begin{array}{l}204.5 \\
(1.21)\end{array}$ & $\begin{array}{l}67.5 \\
(1.48)\end{array}$ & $\begin{array}{c}11770 \\
(8839-15654)\end{array}$ \\
\hline 33 & Delaware & $\begin{array}{l}250 \\
(\mathrm{P})\end{array}$ & 2 June & $\begin{array}{l}40.5 \\
(0.61)\end{array}$ & $\begin{array}{c}0.371 \\
(0.018)\end{array}$ & $\begin{array}{c}190.2 \\
(0.83)\end{array}$ & $\begin{array}{l}52.9 \\
(0.86)\end{array}$ & $\begin{array}{c}15208 \\
(11421-20226)\end{array}$ \\
\hline 34 & $\begin{array}{l}\text { Piedmont } \\
(935)\end{array}$ & $\begin{array}{l}250 \\
(\mathrm{P})\end{array}$ & 18 May & $\begin{array}{l}29.4 \\
(0.24)\end{array}$ & $\begin{array}{c}0.167 \\
(0.004)\end{array}$ & $\begin{array}{c}203.7 \\
(1.22)\end{array}$ & $\begin{array}{l}67.7 \\
(1.34)\end{array}$ & $\begin{array}{c}5005 \dagger \\
(3453-6725)\end{array}$ \\
\hline 35 & $\begin{array}{l}\text { Pleasant Hill } \\
(344)\end{array}$ & $\begin{array}{l}250 \\
(\mathrm{P})\end{array}$ & 18 May & $\begin{array}{l}30.2 \\
(0.21)\end{array}$ & $\begin{array}{c}0.185 \\
(0.004)\end{array}$ & $\begin{array}{c}205.7 \\
(1.35)\end{array}$ & $\begin{array}{l}73.4 \\
(2.60)\end{array}$ & $\begin{array}{c}7725 \\
(6566-9038)\end{array}$ \\
\hline 36 & Pleasant Hill & $\begin{array}{l}250 \\
(\mathrm{P})\end{array}$ & 2 June & $\begin{array}{l}34.8 \\
(0.34)\end{array}$ & $\begin{array}{c}0.235 \\
(0.008)\end{array}$ & $\begin{array}{l}174.9 \\
(0.88)\end{array}$ & $\begin{array}{l}39.5 \\
(0.67)\end{array}$ & $\begin{array}{c}7743 \\
(6581-9059)\end{array}$ \\
\hline 37 & $\begin{array}{l}\text { Tappan } \\
(951)\end{array}$ & $\begin{array}{l}250 \\
(\mathrm{P})\end{array}$ & 18 May & $\begin{array}{l}29.9 \\
(0.23)\end{array}$ & $\begin{array}{c}0.181 \\
(0.005)\end{array}$ & $\begin{array}{c}196.4 \\
(2.85)\end{array}$ & $\begin{array}{l}61.7 \\
(2.64)\end{array}$ & $\begin{array}{c}2093 \dagger \\
(1612-3615)\end{array}$ \\
\hline 38 & Tappan & $\begin{array}{l}151 \\
\text { (P) }\end{array}$ & 4 June & $\begin{array}{l}33.7 \\
(0.35)\end{array}$ & $\begin{array}{c}0.188 \\
(0.015)\end{array}$ & $\begin{array}{c}170.1 \\
(3.43)\end{array}$ & $\begin{array}{l}39.6 \\
(2.48)\end{array}$ & $\begin{array}{c}1650 \dagger \\
(1270-2850)\end{array}$ \\
\hline \multicolumn{9}{|l|}{1994} \\
\hline 40 & $\begin{array}{l}\text { Caesar Creek } \\
(1146)\end{array}$ & $\begin{array}{l}250 \\
(\mathrm{~S})\end{array}$ & 18 May & $\begin{array}{l}28.1 \\
(0.29)\end{array}$ & $\begin{array}{c}0.158 \\
(0.006)\end{array}$ & $\begin{array}{l}220.1 \\
(0.58)\end{array}$ & $\begin{array}{l}83.1 \\
(0.72)\end{array}$ & $\begin{array}{c}5950 \\
(4626-7433)\end{array}$ \\
\hline 41 & $\begin{array}{l}\text { Charles Mill } \\
(547)\end{array}$ & $\begin{array}{l}250 \\
(\mathrm{P})\end{array}$ & 18 May & $\begin{array}{l}26.3 \\
(0.17)\end{array}$ & $\begin{array}{c}0.115 \\
(0.002)\end{array}$ & $\begin{array}{c}228.0 \\
(0.79)\end{array}$ & $\begin{array}{l}92.8 \\
(1.03)\end{array}$ & $\begin{array}{c}3243 \\
(2157-5031)\end{array}$ \\
\hline 42 & $\begin{array}{l}\text { Delaware } \\
(526)\end{array}$ & $\begin{array}{l}250 \\
(\mathrm{~S})\end{array}$ & 18 May & $\begin{array}{l}30.7 \\
(0.24)\end{array}$ & $\begin{array}{c}0.202 \\
(0.005)\end{array}$ & $\begin{array}{c}227.2 \\
(1.12)\end{array}$ & $\begin{array}{l}93.7 \\
(1.77)\end{array}$ & $\begin{array}{c}3209 \dagger \\
(2439-3984)\end{array}$ \\
\hline 43 & $\begin{array}{l}\text { Pleasant Hill } \\
(344)\end{array}$ & $\begin{array}{l}250 \\
(\mathrm{P})\end{array}$ & 18 May & $\begin{array}{l}31.3 \\
(0.29)\end{array}$ & $\begin{array}{c}0.210 \\
(0.006)\end{array}$ & $\begin{array}{l}237.8 \\
(0.88)\end{array}$ & $\begin{array}{c}111.5 \\
(1.36)\end{array}$ & $\begin{array}{c}4681 \\
(2961-7376)\end{array}$ \\
\hline 44 & $\begin{array}{l}\text { Tappan } \\
(951)\end{array}$ & $\begin{array}{l}250 \\
(\mathrm{~S})\end{array}$ & 18 May & $\begin{array}{l}31.6 \\
(0.28)\end{array}$ & $\begin{array}{c}0.262 \\
(0.007)\end{array}$ & $\begin{array}{c}208.3 \\
(1.71)\end{array}$ & $\begin{array}{l}71.6 \\
(1.91)\end{array}$ & $\begin{array}{c}4720 \dagger \\
(2549-6608)\end{array}$ \\
\hline 45 & Piedmont & $\begin{array}{l}250 \\
(\mathrm{P})\end{array}$ & 18 May & $\begin{array}{l}30.4 \\
(0.29)\end{array}$ & $\begin{array}{c}0.224 \\
(0.006)\end{array}$ & $\begin{array}{c}216.1 \\
(1.76)\end{array}$ & $\begin{array}{l}84.3 \\
(2.09)\end{array}$ & $\begin{array}{c}6486 \dagger \\
(3113-9859)\end{array}$ \\
\hline & $\begin{array}{l}\text { Griggs } \ddagger \\
(148)\end{array}$ & $\begin{array}{l}782 \\
(\mathrm{P})\end{array}$ & 25 May & $\begin{array}{l}28.4 \\
(0.15)\end{array}$ & $\begin{array}{c}0.133 \\
(0.003)\end{array}$ & $\begin{array}{c}211.3 \\
(4.29)\end{array}$ & $\begin{array}{l}83.7 \\
(5.60)\end{array}$ & $\begin{array}{c}116 \dagger \\
(45-182)\end{array}$ \\
\hline
\end{tabular}


TABLE 1. Continued.

\begin{tabular}{|c|c|c|c|c|c|c|c|c|}
\hline \multirow[b]{2}{*}{$\begin{array}{l}\text { Year } \\
\text { ID no. }\end{array}$} & \multirow[b]{2}{*}{ Reservoir (ha) } & \multirow{2}{*}{$\begin{array}{c}\text { No. } \\
\text { stocked/ } \\
\text { ha } \\
\text { (method) }\end{array}$} & \multirow[b]{2}{*}{ Stock date } & \multicolumn{4}{|c|}{ Mean sizes (1 SE) } & \multirow[b]{2}{*}{$\begin{array}{l}\text { Fall population } \\
\text { estimate } \\
(95 \% \mathrm{CI})\end{array}$} \\
\hline & & & & $\begin{array}{l}\text { Stock } \\
\text { length } \\
(\mathrm{mm})\end{array}$ & $\begin{array}{l}\text { Stock } \\
\text { mass } \\
(\mathrm{g})\end{array}$ & $\begin{array}{c}1 \text { Oct } \\
\text { length }(\mathrm{mm})\end{array}$ & $\begin{array}{l}1 \text { Oct mass } \\
(\mathrm{g})\end{array}$ & \\
\hline \multicolumn{9}{|l|}{1994} \\
\hline \multirow[t]{5}{*}{45} & O’Shaughnessy & 684 & 25 May & 28.1 & 0.139 & 241.7 & 124.5 & $6084 \dagger$ \\
\hline & $(336)$ & (S) & & $(0.51)$ & $(0.007)$ & $(1.09)$ & & $(2434-9734)$ \\
\hline & Burr Oak $\ddagger$ & $\begin{array}{l}600 \\
(\mathrm{~S})\end{array}$ & 25 May & $\begin{array}{l}33.8 \\
(0.32)\end{array}$ & 0.301 & 201.9 & 61.6 & $\begin{array}{c}161 \dagger \\
(90-232)\end{array}$ \\
\hline & Logan & 635 & 25 May & 33.6 & 0.226 & 132.7 & 16.3 & $103 \dagger$ \\
\hline & $(162)$ & (P) & & $(0.24)$ & $(0.005)$ & $(2.22)$ & $(0.91)$ & $(52-148)$ \\
\hline
\end{tabular}

$\dagger$ Population estimate formulated using a regression model, no./ha ${ }^{3}=0.9763\left(\log _{10} \mathrm{CPH}\right)-0.424$, developed by M. R. Austin (Ohio Division of Wildlife, District 5, Xenia, Ohio).

\$ Prey availability was not sampled, and stocking density was $>2$ times that for other reservoirs; thus these reservoirs were never included on figures.

Identifiable fish prey with complete backbones were measured (nearest millimeter); their wet mass was estimated using backbone length-mass equations from $\mathrm{J}$. M. Dettmers (Illinois Natural History Survey, Alton, Illinois, unpublished data) and Wahl (1988). To evaluate YOY gizzard shad vulnerability to saugeye predation in fall, we determined the proportion of saugeye to which $50 \%$ of the gizzard shad population was available by comparing distributions of saugeye gapes and gizzard shad depths. Gape (greatest vertical dimension when maxillae provided strong resistance, but were not hyperextended) was measured for saugeye collected from Pleasant Hill Reservoir and Caesar Creek Lake in fall 1993 and 1994. Saugeye total length (in millimeters) was converted to gape (in millimeters) using the equation: gape $=0.1132$ (length) $+1.9971\left(r^{2}=\right.$ $0.94, P<0.0001$, length range $98-263 \mathrm{~mm}, N=135$; Donovan et al. 1996). Because the greatest dimension of gizzard shad is depth, we converted lengths of YOY gizzard shad collected during the first 2 wk of October in 1993 and 1994 in Pleasant Hill Reservoir to depth using the following equations (where depth and TL are both measured in millimeters): for gizzard shad $<100$ $\mathrm{mm}$, depth $=0.279$ (TL) -0.737 (J. M. Dettmers, Illinois Natural History Survey, Alton, Illinois, unpublished data); for gizzard shad $\geq 100 \mathrm{~mm}$, depth $=$ 0.298(TL) - 0.536 (Lawrence 1960).

Because saugeye continue to grow during October in Ohio reservoirs, although at a greatly reduced rate (Johnson 1981), and fall saugeye size estimates were attained during $2 \mathrm{wk}$ across reservoirs (a period equal to the stock date treatment in 1993), we adjusted fall saugeye size estimates to estimate mass on 1 October. In reservoirs in which we were able to estimate saugeye growth rates in the fall (i.e., reservoirs with $>50$ recaptures on each circuit of the mark-recapture population estimate), we estimated the growth to 1 October by adjusting individual saugeye sizes by the fall growth rate times number of days (range: $0-8$ ) before or after 1 October that the saugeye was collected. Mean fall growth rate across all reservoirs within each year was applied for reservoirs with insufficient recaptures.

\section{1 and 1992 reservoir monitoring}

Similar data to those collected from reservoirs in 1993 and 1994 were collected during $1991(N=4$ reservoirs; reported in Stahl et al. 1996; Table 1) and $1992(N=8$; reported in Stahl et al. 1993; Table 1). Sampling for zooplankton, larval fish, and age-0 saugeye were as described for the 1993 reservoir experiment, with the following exceptions. In 1991, zooplankton and larval fish were sampled weekly during 15 April-30 June, and at least once every 2 wk through September (Stahl et al. 1996). In 1992, zooplankton and larval fish were sampled weekly from $1 \mathrm{wk}$ before stocking to $2 \mathrm{wk}$ after stocking in all reservoirs except

TABLE 2. Characteristics of prey availability and saugeye growth and survival from the pond experiment conducted during 6 May-2 June 1994. Zooplankton includes cyclopoids, Daphnia, and calanoids. Values are means (with 1 SE reported in parentheses). See Materials and methods for sample sizes.

\begin{tabular}{|c|c|c|c|c|c|c|c|}
\hline \multirow[b]{4}{*}{ Pond treatment } & \multicolumn{3}{|c|}{ Gizzard shad } & & & \multirow{2}{*}{\multicolumn{2}{|c|}{ Saugeye }} \\
\hline & \multirow{3}{*}{$\begin{array}{l}\text { Peak density } \\
\quad\left(\text { no. } / \mathrm{m}^{3}\right)\end{array}$} & \multirow{3}{*}{$\begin{array}{c}\text { Final } \\
\text { density } \\
\left(\text { no. } / \mathrm{m}^{3}\right)\end{array}$} & \multirow{3}{*}{$\begin{array}{c}\text { Final } \\
\text { length } \\
(\mathrm{mm})\end{array}$} & \multirow{2}{*}{\multicolumn{2}{|c|}{ Zooplankton (no./L) }} & & \\
\hline & & & & & & \multirow{2}{*}{$\begin{array}{l}\text { Wet mass } \\
\text { increase }(\mathrm{g})\end{array}$} & \multirow{2}{*}{$\begin{array}{c}\text { Surviva } \\
(\%)\end{array}$} \\
\hline & & & & Initial & Final & & \\
\hline $\begin{array}{l}\text { Saugeye and gizzard shad } \\
(N=3)\end{array}$ & $\begin{array}{l}17.7 \\
(6.2)\end{array}$ & $\begin{array}{l}11.7 \\
(5.1)\end{array}$ & $\begin{array}{c}6.4 \\
(0.2)\end{array}$ & $\begin{array}{l}234.7 \\
(81.5)\end{array}$ & $\begin{array}{c}203.9 \\
(135.1)\end{array}$ & $\begin{array}{l}1.39 \\
(0.05)\end{array}$ & $\begin{array}{l}73.7 \\
(1.9)\end{array}$ \\
\hline $\begin{array}{l}\text { Saugeye only } \\
\quad(N=2)\end{array}$ & & & & $\begin{array}{l}216.2 \\
(15.8)\end{array}$ & $\begin{array}{c}74.3 \\
(12.6)\end{array}$ & $\begin{array}{l}1.37 \\
(0.18)\end{array}$ & $\begin{array}{l}76.4 \\
(12.5)\end{array}$ \\
\hline $\begin{array}{l}\text { Gizzard shad only } \\
\quad(N=1)\end{array}$ & 156.8 & 46.7 & $\begin{array}{c}9.1 \\
(0.8)\end{array}$ & 175.3 & 30.2 & & \\
\hline
\end{tabular}




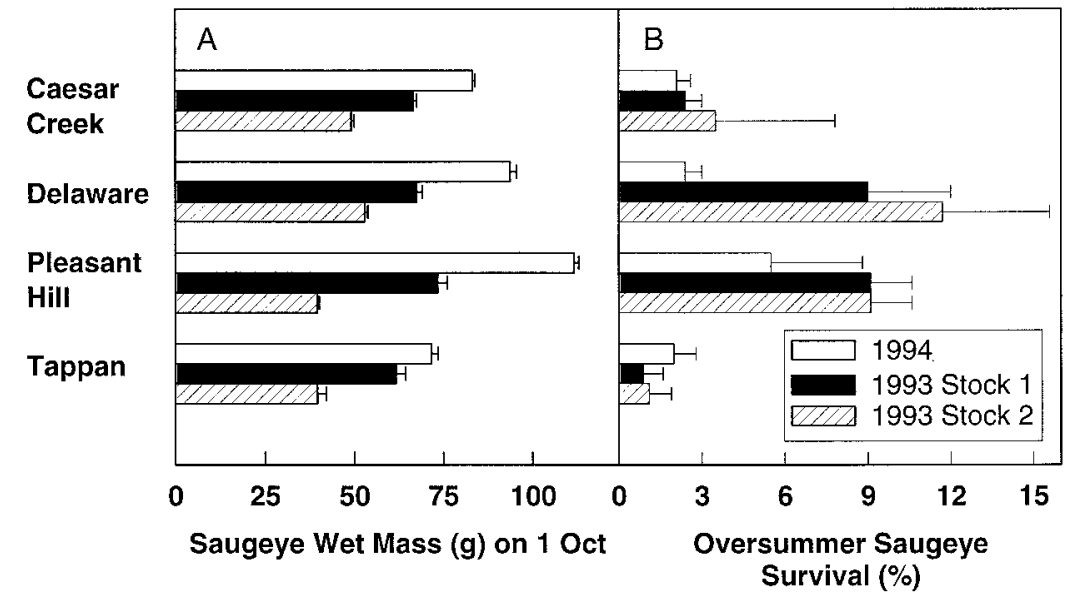

FIG. 1. (A) Mean (and $1 \mathrm{SE}$ ) wet mass on 1 October and (B) oversummer survival (with upper 95\% CI) of saugeye from the 1993 and 1994 stocking experiments in Ohio reservoirs. See Table 1 for initial conditions.

Delaware and Pleasant Hill, which were sampled twice a week during the $2 \mathrm{wk}$ before stocking to $3 \mathrm{wk}$ after stocking, and at least once every 2 wk through October (Stahl et al. 1993).

\section{RESULTS}

\section{Enclosure experiment}

Switch to piscivory.-Without saugeye, mean $( \pm 1$ SE) number of larval gizzard shad surviving for $24 \mathrm{~h}$ in enclosures with 20 gizzard shad was $17.8 \pm 0.5$ larval gizzard shad $(N=4)$. Recovery of gizzard shad from enclosures with saugeye dismantled at hour 12 was $0 \pm 0,1 \pm 0$, and $3 \pm 1.5$ larval gizzard shad for treatments of 5, 10, and 20 larval gizzard $\mathrm{shad} / \mathrm{m}^{3}$. Thus, saugeye in all treatments with larval gizzard shad switched to piscivory within $12 \mathrm{~h}$.

Growth.-Saugeye growth did not differ among larval gizzard shad densities, but saugeye in all treatments with larval gizzard shad grew faster than those with only zooplankton (one-way ANOVA, $P<0.0001$, Tukey hsd, $\alpha=0.005)$. Wet mass of saugeye at the end of $13 \mathrm{~d}$ was ordered across larval gizzard shad densities (no. gizzard shad $\left./ \mathrm{m}^{3}\right): 0.46 \pm 0.062(0$, zooplankton only), $1.02 \pm 0.039$ (5), $1.08 \pm 0.04$ (10), and $1.22 \pm$ 0.082 (20) g. Mean crustacean zooplankton density excluding rotifers (range 278.6-484.6 individuals/L) did not differ among treatments on day 0 (one-way ANOVA, $P=0.279$ ). Final zooplankton densities did differ, ranging from $14.3 \pm 6.7$ individuals/L in enclosures with 20 larval gizzard shad to $150.4 \pm 29.8$ individuals/L in enclosures without larval gizzard shad (one-way ANOVA, $P=0.022$ ). Mean density of those taxa commonly eaten by saugeye, cyclopoids, Daphnia, and calanoids (Fielder 1992, Stahl and Stein 1994), was $41.2 \pm 7.9$ individuals/ $\mathrm{L}$ on day 0 and $3.2 \pm 1.1$ individuals/L on day 13 , and did not differ among treatments (one-way ANOVAs, day $0, P=0.19$; day 13, $P=0.72)$.

\section{Pond experiment}

Larval gizzard shad were first captured on 11 May, $5 \mathrm{~d}$ after saugeye stocking. In ponds with saugeye, peak densities of 10-30 larval gizzard $\mathrm{shad} / \mathrm{m}^{3}$ occurred $12-$ $15 \mathrm{~d}$ after stocking. Whereas, without saugeye, a peak density of 156.8 larval gizzard shad $/ \mathrm{m}^{3}$ occurred $9 \mathrm{~d}$ after stocking (Table 2). Final larval gizzard shad length and density were greater in the pond without saugeye (length, $t$ test, $P<0.0001$; Table 2). Mean initial and final density of preferred zooplankton taxa ranged from 30.2 to $234.7 / \mathrm{L}$ and did not differ among saugeye treatments ( $t$ tests, all $P$ values $>0.43$; Table 2).

Mass of saugeye stomach contents was greater in ponds with gizzard shad (mean dry diet mass/saugeye dry mass, $0.109 \pm 0.052 \mathrm{~g}$ ) than in ponds with zooplankton and macrobenthic prey only $(0.052 \pm 0.005$ $\mathrm{g} ; t$ test, $P<0.0001)$ across all sample dates. However, during the overlap between larval gizzard shad and saugeye, only 2 of 54 saugeye contained larval gizzard shad. Greater diet mass of saugeye in ponds with larval gizzard shad derived from greater consumption of macrobenthos $(0.095 \pm 0.014 \mathrm{~g})$ than in ponds with zooplankton and macrobenthic prey only $(0.034 \pm 0.005$ $\mathrm{g}$; $t$ test, $P<0.0001)$. After $30 \mathrm{~d}$, saugeye wet mass increase and survival did not differ between treatments ( $t$ tests: wet mass, $P=0.93$; survival, $P=0.8$; Table 2).

\section{3 reservoir experiment}

On 1 October 1993, stock-1 saugeye weighed more than stock-2 saugeye in all reservoirs ( $t$ tests, all $P<$ 0.0001 ; Fig. 1A) with mean mass of stock 1 (61.7$73.4 \mathrm{~g}) 68 \pm 6 \%$ greater than that of stock 2 (39.5$52.9 \mathrm{~g})$. Mean mass of stock-1 saugeye differed among reservoirs (one-way ANOVA, $P=0.034$ ); however, multiple comparisons tests (Tukey hsd, $\alpha=0.05$ ) were not significant. 


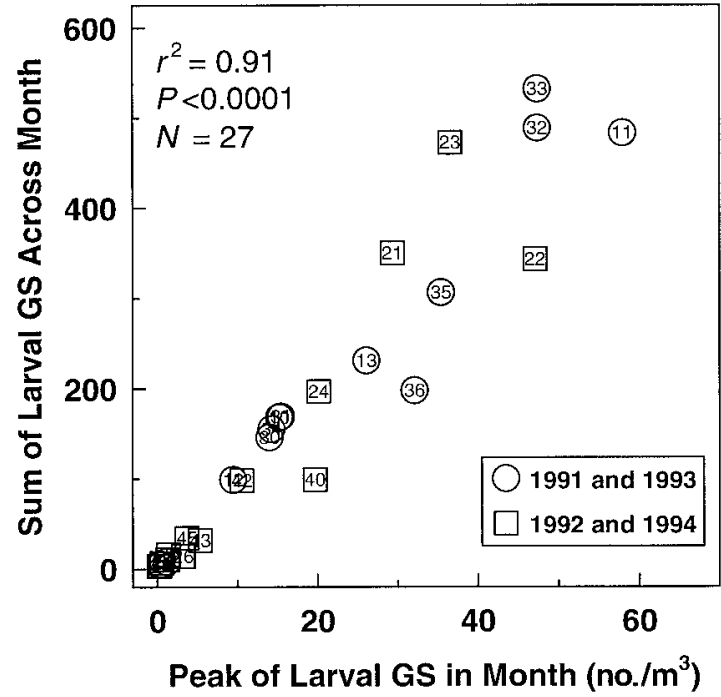

FIG. 2. Sum of larval gizzard shad (GS) densities (integrated values under the density curve) as a function of their peak densities in the months around saugeye stocking in Ohio reservoirs during 1991-1994. Codes for reservoirs with $<10$ larval gizzard $\mathrm{shad} / \mathrm{m}^{3}$ are $12,20,25,26,27,37,38,41,43$, 44, and 45. For reservoir codes, see Table 1.

Regardless of stock, oversummer saugeye survival ranged from 0.9 to $11.7 \%$; stock- 2 survival was equal to or greater than that of stock 1 in all reservoirs (Fig. 1B). Almost all saugeye mortality occurred before a summer population estimate in Pleasant Hill Reservoir. Survival of the combined stocks to 5 August was $9.6 \%$ $(7.0-13.2 \%, 95 \% \mathrm{CI})$ and did not differ from the survival of either stock to 1 October $(9.1 \%, 7.7-10.6 \%$, 95\% CI for both stocks).

Early-season prey and saugeye success.-To assess how availability of larval gizzard shad (which contributes $>90 \%$ of total larval fish by density) influences saugeye success, we regressed saugeye wet mass on 1 October and oversummer survival against two measures of gizzard shad abundance. First, because saugeye can immediately convert to piscivory (Stahl and Stein 1994; enclosure experiment, this study), we regressed saugeye mass on 1 October and oversummer survival against gizzard shad density on the date of stocking. Second, we regressed our estimates of saugeye success against peak density within a reservoir because peak gizzard shad density in Ohio reservoirs in 1991-1994 was correlated strongly with the sum of the daily estimates of gizzard shad density during the month surrounding stock date (Fig. 2). For both stocks across all reservoirs in 1993, gizzard shad density on stocking dates ranged from 0.4 to 35.4 larval gizzard $\mathrm{shad} / \mathrm{m}^{3}$; peak density ranged from 1.2 to 47.4 larval gizzard $\mathrm{shad} / \mathrm{m}^{3}$. Peak density occurred within $5 \mathrm{~d}$ of stock-1 addition (18-19 May), except in Caesar Creek Lake in which the density on stocking date was 14 larval gizzard $\mathrm{shad} / \mathrm{m}^{3}$, and peak density (15.4 larval gizzard $\mathrm{shad} / \mathrm{m}^{3}$ ) occurred 8 June.
Across all reservoirs in 1993, gizzard shad density on the stock date positively influenced mass of saugeye on 1 October for stock $1\left(r^{2}=0.88, P=0.017, N=\right.$ $5)$, but not for stock $2\left(r^{2}=0.64, P=0.202, N=4\right)$. For all reservoirs in 1993, peak density did not influence saugeye growth to 1 October (Table 3). Oversummer saugeye survival was correlated with gizzard shad density on stocking date for stock $1\left(r^{2}=0.80, P=\right.$ $0.04, N=5)$, but not for stock $2\left(r^{2}=0.64, P=0.197\right.$, $N=4)$. Peak density positively influenced saugeye oversummer survival for both stocks (Table 3 ).

As a measure of zooplankton prey available to newly stocked saugeye, we summed daily biomass estimates of preferred zooplankton taxa for 2 wk after stocking. Zooplankton availability did not influence growth or survival of saugeye in either cohort through fall (Table $3)$.

\section{4 reservoir experiment}

Mean wet mass of saugeye on 1 October ranged from 61.6 to $124.5 \mathrm{~g}$, except in Lake Logan $(16.3 \pm 0.91 \mathrm{~g}$; Table 1); oversummer survival ranged from 0.1 to $5.5 \%$. Neither point- nor scatter-stocked saugeye differed in mean $( \pm 1 \mathrm{SE})$ survival $(2.2 \pm 1.0 \%$ vs. $1.8 \pm$ $0.4 \%$, respectively) or mean growth $(78.3 \pm 17.0 \mathrm{~g}$ vs. $87.0 \pm 11.1 \mathrm{~g}$, respectively) to 1 October ( $t$ tests, $P \mathrm{~s}$ $>0.6, N=10)$.

Early-season prey and saugeye success.-Density was $<2$ larval gizzard $\mathrm{shad} / \mathrm{m}^{3}$ in all reservoirs when saugeye were stocked (18 or 25 May) in 1994. In the six reservoirs sampled for prey availability, peak density $\left(0.3-19.7\right.$ larval gizzard $\left.\mathrm{shad} / \mathrm{m}^{3}\right)$ occurred $\geq 2 \mathrm{wk}$ after saugeye stocking and did not influence stocking success (Table 3).

Because YOY gizzard shad can regulate zooplankton (Dettmers and Stein 1992), low larval gizzard shad densities in 1994 allowed us to assess the influence of zooplankton near stocking on saugeye success. The sum of the daily estimates of preferred zooplankton biomass during the $2 \mathrm{wk}$ after stocking did not influence saugeye mass on 1 October or oversummer survival (Table 3).

\section{Broadscale field patterns of saugeye success}

We begin by reviewing our field data with the express purpose of identifying those features of the environment, specifically mass at stocking, days in reservoir, and prey resources that could explain broadscale patterns of oversummer growth and survival of saugeye. Across all years, saugeye mass at stocking did not influence oversummer survival $\left(r^{2}=0.06, P=0.346, N\right.$ $=27$ ), but was correlated negatively with saugeye mass on 1 October $\left(r^{2}=-0.25, P=0.008, N=27\right)$. Mean saugeye mass on 1 October was correlated positively with days of reservoir residence in 1993 (Table 3) and across all reservoirs and years (Table 3). Zooplankton biomass appeared to influence saugeye mass on $1 \mathrm{Oc}-$ tober across all years (Table 3 ). However, because the 
TABLE 3. Results from simple linear regressions of saugeye mass on 1 October and oversummer survival vs. measures of prey density, number of days in each reservoir, and timing of larval gizzard shad peaks for 1993 (two stocks), 1994, and 1991-1994 combined.

\begin{tabular}{|c|c|c|c|c|c|c|c|}
\hline \multirow{2}{*}{$\begin{array}{l}\text { Saugeye response } \\
\text { variable }\end{array}$} & \multirow[b]{2}{*}{ Independent variable } & \multicolumn{3}{|c|}{1993 Stock 1} & \multicolumn{3}{|c|}{1993 Stock 2} \\
\hline & & $N$ & $r^{2}$ & $P$ & $N$ & $r^{2}$ & $P$ \\
\hline \multirow[t]{4}{*}{ Mass on 1 Oct } & Peak gizzard shad density & 5 & 0.45 & 0.22 & 4 & 0.31 & 0.44 \\
\hline & Sum zooplankton biomass & 5 & $<0.0001$ & 0.93 & 4 & 0.09 & 0.69 \\
\hline & Time in reservoir & $9 \dagger$ & 0.85 & $<0.001$ & & & \\
\hline & $\begin{array}{l}\text { No. days stocked before/after } \\
\text { peak gizzard shad density }\end{array}$ & $7+t$ & 0.29 & 0.22 & & & \\
\hline \multirow[t]{4}{*}{ Oversummer survival } & Peak gizzard shad density & 5 & 0.92 & $<0.001$ & 4 & 0.98 & $<0.01$ \\
\hline & Sum zooplankton biomass & 5 & -0.06 & 0.69 & 4 & -0.11 & 0.66 \\
\hline & Time in reservoir & $9 \dagger$ & 0.03 & 0.66 & & & \\
\hline & $\begin{array}{l}\text { No. days stocked before/after } \\
\text { peak gizzard shad density }\end{array}$ & $7 \dagger+$ & 0.41 & 0.12 & & & \\
\hline
\end{tabular}

$\dagger$ Stocks 1 and 2 were combined to yield a single value because individual stocks were added to reservoirs on a single day.

\$ Number of reservoirs was smaller for number of days stocked before/after peak gizzard shad density because only reservoirs with at least 5 larval gizzard shad $/ \mathrm{m}^{3}$ were included.

$\S$ Saugeye were added on 18 May to all reservoirs stocked at a density of 250 individuals/ha in 1994 .

linear relationship explained so little of the variability and because zooplankton biomass did not influence saugeye mass on 1 October in any individual year, this may be a spurious relationship. Across all years, zooplankton biomass did not influence saugeye oversummer survival. For all reservoirs across all years, peak larval gizzard shad density did not influence saugeye mass on 1 October (Table 3 ). In contrast, saugeye oversummer survival did correlate with peak density (Table 3). Interestingly, peak densities occurred earlier in 1991 and 1993 than in 1992 and 1994, probably due to differences in spring warming patterns. In Pleasant Hill and Delaware reservoirs, peak densities of gizzard shad occurred on 20 May $\pm 1 \mathrm{~d}$ (mean $\pm 1 \mathrm{SE}, N=4)$ in 1991 and 1993 (warm years), whereas peaks occurred on 5 June $\pm 4 \mathrm{~d}(N=4)$ in 1992 and 1994 (cool years). Oversummer survival was correlated strongly with peaks in warm years, but not cool ones (Fig. 3).

We now turn to the four reservoirs most intensively sampled during 1991-1994 (Caesar Creek, Delaware, Pleasant Hill, and Tappan) to further define our broadscale field patterns. In these reservoirs, saugeye were larger on 1 October in 1992 and 1994 (cool years) than in 1991 and 1993 (warm years; ANOVA, blocked by reservoir, Tukey hsd, $\alpha=0.05, P=0.0005)$. Survival differed among all years (ANOVA, blocked by reservoir, $P=0.014$ ), but in tests of multiple comparisons, only 1991 survival was higher than other years (Tukey hsd, $\alpha=0.05$ ). For comparisons within 1993 (stocks 1 and 2) and to 1994, saugeye mass on 1 October was greater in 1994, followed by stock 1 of 1993 , then stock 2 (ANOVA, blocked by reservoir, Tukey hsd, $\alpha=0.05$, $P=0.0019$; see Fig. 1A), and survival tended to be greater in 1993 than 1994, but did not differ statistically (ANOVA, blocked by reservoir, $P=0.259$; see Fig. 1B).

\section{Ontogeny of saugeye}

We now use our data, gathered during 1993-1994 via experiments and field research, to provide a syn- thetic, explanatory hypothesis of saugeye success using their ontogeny as an organizing framework. In so doing, we have divided the ontogeny of saugeye into two periods: (1) stocking to $\sim 1$ August and (2) 1 August1 October. Two reservoirs, Delaware and Pleasant Hill, provide the majority of the data; data from our other study reservoirs, though not included herein, were nevertheless consistent with our conclusions.

Stocking to 1 August.-Saugeye growth poststocking through July was similar among the two stocks in 1993 and the single stock in 1994 (Fig. 4A, B). Saugeye primarily consumed zooplankton and chironomids immediately after stocking; conversion to piscivory was delayed 1-5 wk (also reported in Stahl et al. 1996). In reservoirs with adequate recovery of saugeye to assess diet composition ( $N \geq 5$ saugeye/date), we quantified the date saugeye switched to piscivory $(\geq 50 \%$ with fish prey) in 1993 and 1994. Delay to piscivory was longer in $1994(27 \pm 1.7 \mathrm{~d}, N=4)$ than in stock 1 $(17.5 \pm 1.5 \mathrm{~d}, N=4)$ and stock $2(9.7 \pm 2.3 \mathrm{~d}, N=$ 3 ) of 1993 (one-way ANOVA, Tukey hsd, $\alpha=0.05$, $P=0.001$ ). Dry prey mass per dry saugeye mass from stocking to 31 June did not differ between either stock in any reservoir in 1993 ( $t$ tests, $P$ s $>0.5$ ), or in 1994 (one-way ANOVAs, $P$ s $>0.3$ ).

Although stock-1 saugeye were larger than stock-2 saugeye at the end of July ( $t$ test of total length, Delaware Reservoir, $P=0.03$; Pleasant Hill Reservoir, $P$ $<0.0001$; see Fig. 5A for length distributions from Pleasant Hill Reservoir), these differences were small (Delaware Reservoir-stock 1 , mean $\pm 1 \mathrm{SE}, 141.3 \pm$ $2.5 \mathrm{~mm}$, stock 2, $134.4 \pm 2.0 \mathrm{~mm}$; Pleasant Hill Reservoir—stock $1,146 \pm 0.8 \mathrm{~mm}$, stock $2,133.9 \pm 0.9$ mm; Fig. 4C, D). For Pleasant Hill Reservoir, saugeye from 1994 were larger than stock 2 of 1993, but did not differ from stock 1 (one-way ANOVA, Tukey hsd, $\alpha=0.05, P<0.0001$; Fig. 4C). In Pleasant Hill Reservoir on 5 August 1993, at least $85 \%$ of saugeye from both stocks had fish in their diet (stock $1, N=36$; stock 
TABLE 3. Continued.

\begin{tabular}{cccccccc}
\hline \hline & 1994 & & & \multicolumn{3}{c}{$1991-1994$} \\
\cline { 1 - 2 } \cline { 5 - 7 }$N$ & $r^{2}$ & $P$ & & $N$ & $r^{2}$ & $P$ \\
\hline 6 & $<0.001$ & 0.95 & & 27 & -0.08 & 0.16 \\
6 & 0.26 & 0.30 & & 27 & 0.18 & $<0.05$ \\
$\S$ & & & & 27 & 0.46 & $<0.0001$ \\
& & & & & \\
6 & 0.13 & 0.64 & & $19 \neq$ & 0.44 & $<0.001$ \\
6 & 0.02 & 0.78 & & 27 & 0.56 & $<0.0001$ \\
6 & 0.10 & 0.55 & & 27 & 0.01 & 0.49 \\
$\S$ & & & & 27 & -0.03 & 0.42 \\
6 & 0.2 & 0.56 & & $19 \ddagger$ & 0.37 & $<0.01$ \\
\hline
\end{tabular}

2, $N=32$ ). On 28 July, most saugeye from Delaware had fish in their diet (stock 1,62\%; stock 2,89\%), but sample sizes were small (stock $1, N=8$; stock $2, N$ $=9)$.

1 August to 1 October.-Saugeye grew fastest to 1 October in 1994, followed by stock 1 of 1993, then stock 2 (one-way ANOVA, Tukey hsd, $\alpha=0.05, P$ s $<0.0001$ for both Pleasant Hill and Delaware reservoirs; Fig. 4A, B). Differences in daily growth rate to fall were small (Fig. 4A, B). Nonetheless, these small differences in growth rate were compounded daily, leading to large differences in saugeye length on 1 October (Fig. 4C, D). In Pleasant Hill and Delaware reservoirs, saugeye length-frequency distributions differed among all stocks during 1993 and 1994 (Kolmogorov-Smirnov, all $P$ s $<0.0001)$. In Pleasant Hill Reservoir, the length-frequency distribution on 1 October was most broad for stock 1 (18 10-mm length classes), less so for stock 2 (14 length classes) and in 1994 (11 length classes; see Fig. 5B for 1993 data). Given the exponential relationship between length and mass, saugeye length differences on 1 October translated into profound differences in mass (Fig. 4E, F). Again, patterns were similar across all reservoirs in 1993 and 1994 (see Fig. 1A and Table 1).

Gizzard shad were larger in fall 1993 (103 \pm 0.5 $\mathrm{mm}, N=154$; early peak density) than in fall 1994 (77.7 $\pm 0.43 \mathrm{~mm}, N=222$; late peak density) in Pleasant Hill Reservoir ( $t$ test, $P<0.0001$ ), probably due to time of spawning. In Pleasant Hill Reservoir, a greater proportion (46\%; Fig. 6B) of stock-1 saugeye than stock-2 saugeye (5\%; Fig. 6A) had gapes larger than the median gizzard shad depth in 1993. In 1994, all saugeye gapes were larger than the median gizzard shad depth (Fig. 6C).

Diets of saugeye in Pleasant Hill Reservoir in fall 1993 and 1994 reflect the ability of saugeye to consume YOY gizzard shad. Across both stocks in 1993, diets of smaller saugeye were less likely to contain fish (Fig. 7A, B). Using logistic regression across both cohorts, we estimated that the length necessary for $75 \%$ piscivory was $202 \mathrm{~mm}(P=0.0001)$. Fifty-three percent of stock-1 saugeye were $\geq 202 \mathrm{~mm}$, as compared to

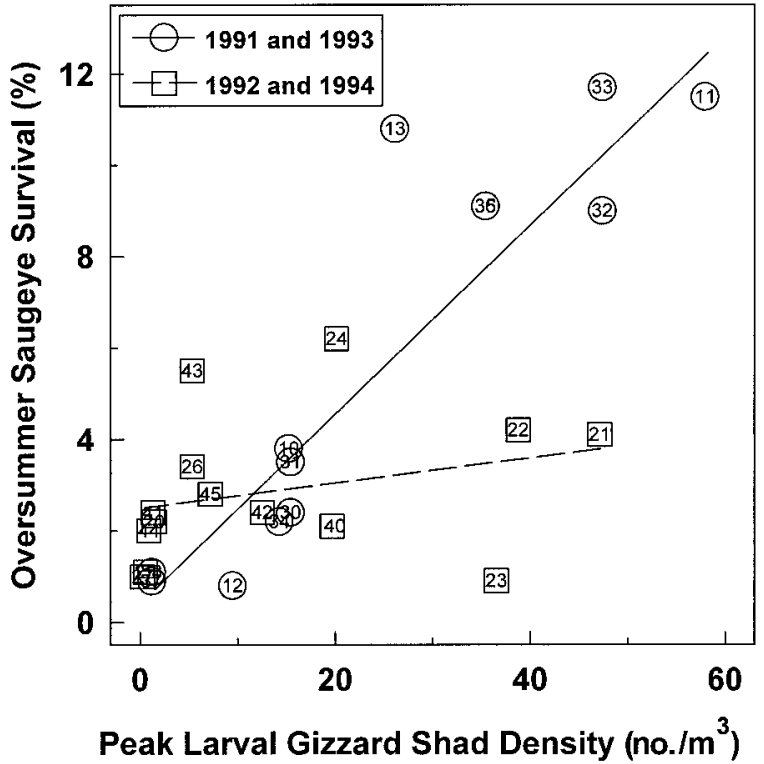

FIG. 3. Oversummer survival of saugeye as a function of peak gizzard shad density for years of early $(1991+1993$ : $\left.r^{2}=0.83, P<0.0001, N=13\right)$ and late $\left(1992+1994: r^{2}\right.$ $=0.11, P=0.236, N=14)$ peaks in Ohio reservoirs. Codes for reservoirs in which peak larval gizzard shad density was $<5$ larval gizzard shad/ $\mathrm{m}^{3}$ are 20, 25, 27, 37, 38, 41, and 44 . Reservoir codes 35 and 36 share the same coordinates. For reservoir codes, see Table 1.

only $10 \%$ for stock- 2 saugeye. To compensate for limited gizzard shad prey, a greater proportion $(31 \%)$ of stock-2 saugeye in Delaware Reservoir consumed nongizzard shad forage fishes, primarily Lepomis spp., than in Pleasant Hill Reservoir (9\%). Alternately, more stock-2 saugeye from Pleasant Hill Reservoir consumed macroinvertebrates $(37 \%)$ than in Delaware Reservoir (1\%). Thus, the greater disparity in mean mass on 1 October between stocks 1 and 2 in Pleasant Hill Reservoir (stock $186 \%$ heavier than stock 2) than in Delaware Reservoir (28\%) may have derived from greater consumption of slower growing forage fishes (also described by Santucci and Wahl [1993] for a centrarchid prey community). In 1994, almost all saugeye in Pleasant Hill Reservoir (95\%) contained fish prey (Fig. 7C).

Growth-survival trade-offs.-During 1991-1994, oversummer saugeye survival and mass on 1 October were unrelated (Fig. 8). To determine the influence of early peaks of gizzard shad in warm years (1991 and 1993) and late peaks in cool years (1992 and 1994), we assessed saugeye growth and survival with respect to the interaction between saugeye stock date and gizzard shad peak within a reservoir across years. Because we were assessing saugeye success relative to variation in timing of gizzard shad peaks, only reservoirs in which peaks $>5$ larval gizzard $\mathrm{shad} / \mathrm{m}^{3}$ occurred were included. When stocked before larval gizzard shad peaks, saugeye consistently survived poorly to fall 
Pleasant Hill Delaware

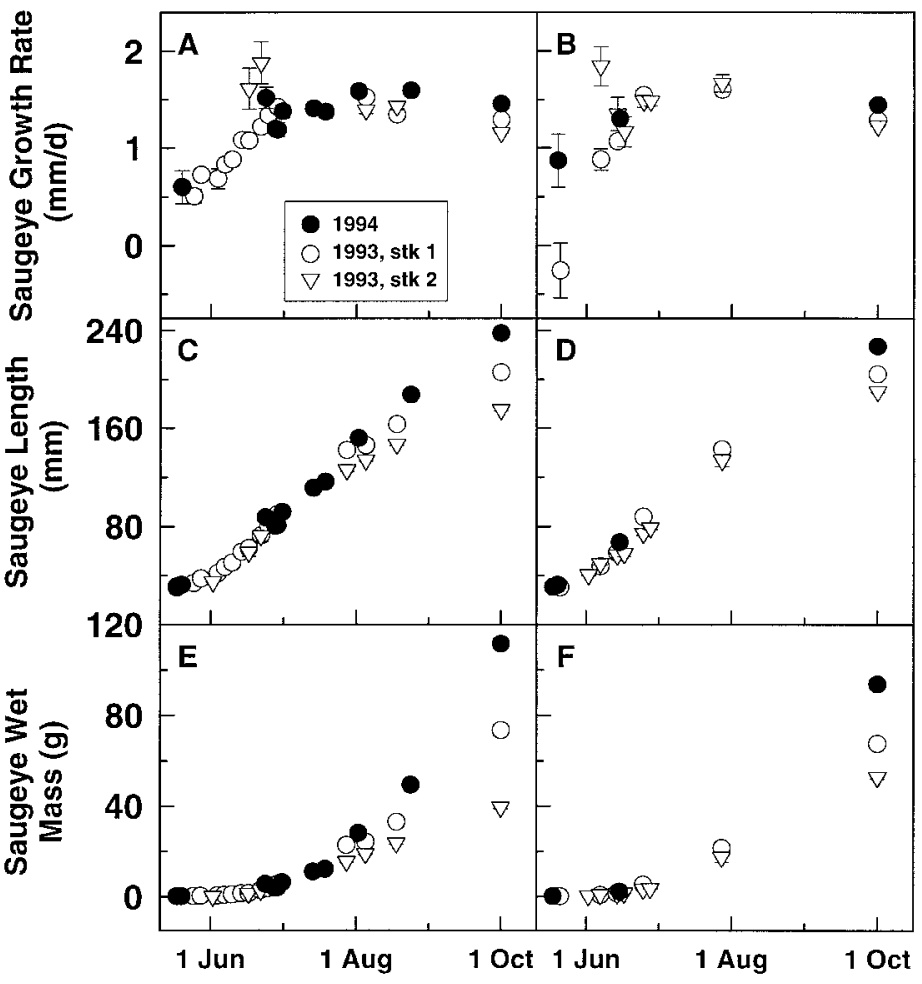

FIG. 4. Mean growth rates $(\mathrm{mm} / \mathrm{d})$, total lengths $(\mathrm{mm})$, and wet mass $(\mathrm{g})$ of saugeye $( \pm 1$ SE) through time in Pleasant Hill and Delaware reservoirs, Ohio, during 1993 and 1994. $N \geq 5$ for all data points. For sizes at stocking, date of stocking, and size on 1 October, see Table 1.
(Fig. 9A). When stocked within \pm 1 wk of the peak, oversummer survival was highly variable. Saugeye stocked $>1$ wk after the peak always enjoyed high survival. In turn, saugeye mass on 1 October declined the later the stock time relative to gizzard shad peaks. Saugeye stocked $>1$ wk before peaks were quite large on 1 October, whereas those stocked $>1$ wk after peaks were quite small (Fig. 9B).

\section{Overwinter survival}

In Pleasant Hill Reservoir, mean size of both stocks did not differ during 1 October 1993-19 April 1994 ( $t$ tests, $P$ s $>0.3$ ). Size distributions did differ (Kolmogorov-Smirnov, $P<0.0001$; Fig. 5C), perhaps deriving from the limited sample in April $(N=197)$. Saugeye as small as $113 \mathrm{~mm}$ survived winter. Because recaptures were few in Delaware Reservoir $(N=41)$, we combined saugeye from both stocks. Mean saugeye size did not differ overwinter ( $t$ test, $P=0.94$ ) and saugeye as small as $167 \mathrm{~mm}$ survived winter.

\section{DisCUSSION}

Our experiments reveal that interactions with age-0 gizzard shad influence saugeye growth and survival through their first year. However, for all reservoir stockings $(N=31)$, mass on 1 October and oversummer survival were unrelated, counter to our initial expectations that (1) larval gizzard shad density influences time to piscivory, (2) piscivory increases saugeye growth, and (3) faster growth improves survival through fall. In fact, how age-0 gizzard shad influence saugeye growth and survival appears to be independent and separated in time, first driving saugeye survival, then growth through fall. In reservoirs with peak density $>5$ larval gizzard $\mathrm{shad} / \mathrm{m}^{3}$, saugeye stocked before peaks were large on 1 October, but survived poorly to fall, whereas saugeye stocked after peaks were small, but enjoyed higher survival. To explore ecological mechanisms underlying these patterns, we track saugeye diet and growth through their first summer in Ohio reservoirs.

\section{Overview with a caveat}

Despite a $>125$-yr history of stocking percids, stocking success remains low and variable with little opportunity for accurately characterizing all sources of mortality (Laarman 1978). Oversummer saugeye survival in Ohio reservoirs ranged from 0 to $11.7 \%$ across all years. As starvation appears unimportant, saugeye persistent in reservoirs have avoided losses due to transportation and handling stress, predation, and outmigration. Because transportation times were short ( $\leq 2$ h), handling/transportation mortality (including assessments to 7-10 d poststocking) is thought to be low (17-32\%, Hurley and Austin 1987; <20\%, Stahl et al. 1996) and unpredictable. As open-ended systems, reservoirs allow outmigration leading to both upstream and tailwater populations (Jernejcic 1986), the extent 


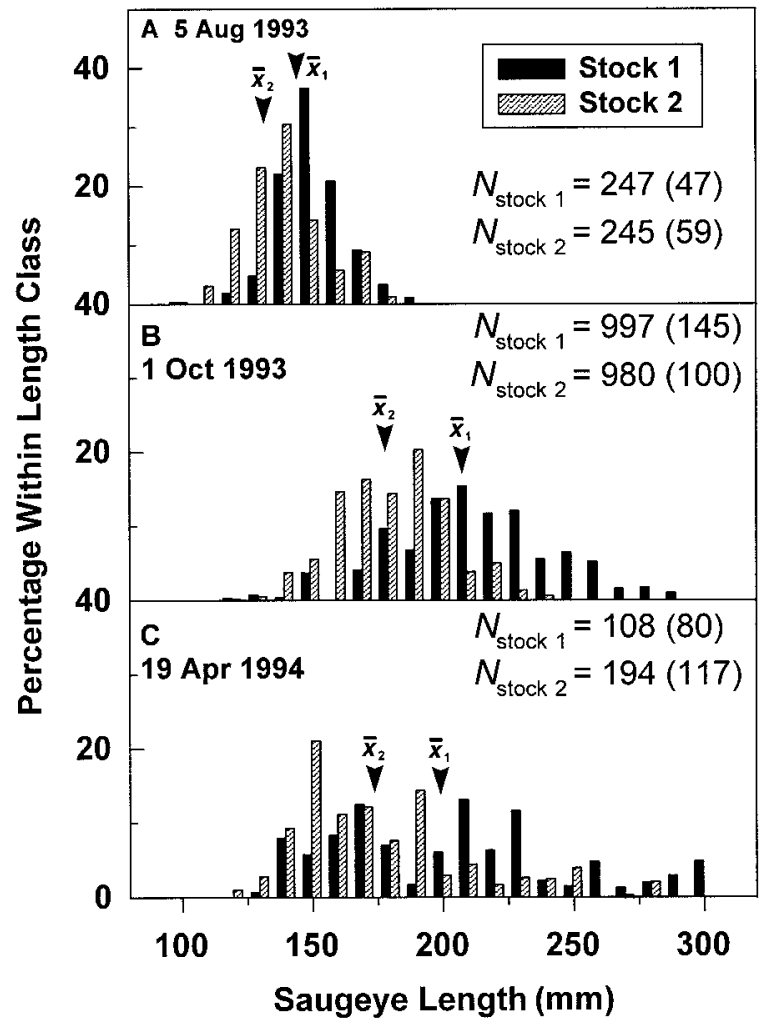

FIG. 5. Length distributions (percentage within each 10 -mm length class) of stock-1 and stock-2 saugeye from Pleasant Hill Reservoir, Ohio on (A) 5 August 1993, (B) 1 October 1993, and (C) 19 April 1994. Number reported for each stock is the total number of each stock collected on each date. Numbers in parentheses are the numbers of saugeye for which protein type was identified. Arrows denote mean sizes of stock-1 and stock-2 saugeye on each sampling date. Saugeye not identified by protein type were randomly assigned to stocks according to the stock 1 : stock 2 ratio within a length class as determined by protein type.

of which is unknown. Widely implicated for other stocked fishes (Stein et al. 1981, B. L. Johnson et al. 1988, Wahl and Stein 1989), predatory losses for Ohio saugeye are generally $<20 \%$ (Stahl et al. 1996). Hence, we view our population estimates as relative, rather than absolute, measures of the severity of mortality forces within a reservoir.

\section{Prey density and saugeye survival: size-independent mechanisms}

By stocking saugeye as fingerlings (30-40 mm), managers circumvent the high mortality characteristic of fishes developing from larvae to fingerlings. Stocked saugeye are beyond the critical endogenous-exogeneous feeding transition ( $\mathrm{Li}$ and Mathias 1982) and beyond that time when larval diet, growth, and predatory vulnerability are so intimately related (Post and Prankevicius 1987, Rice et al. 1987b, Miller et al. 1988, Luecke et al. 1990). Hence, even though oversummer survival only ranged from 0 to $11.7 \%$, saugeye prob-

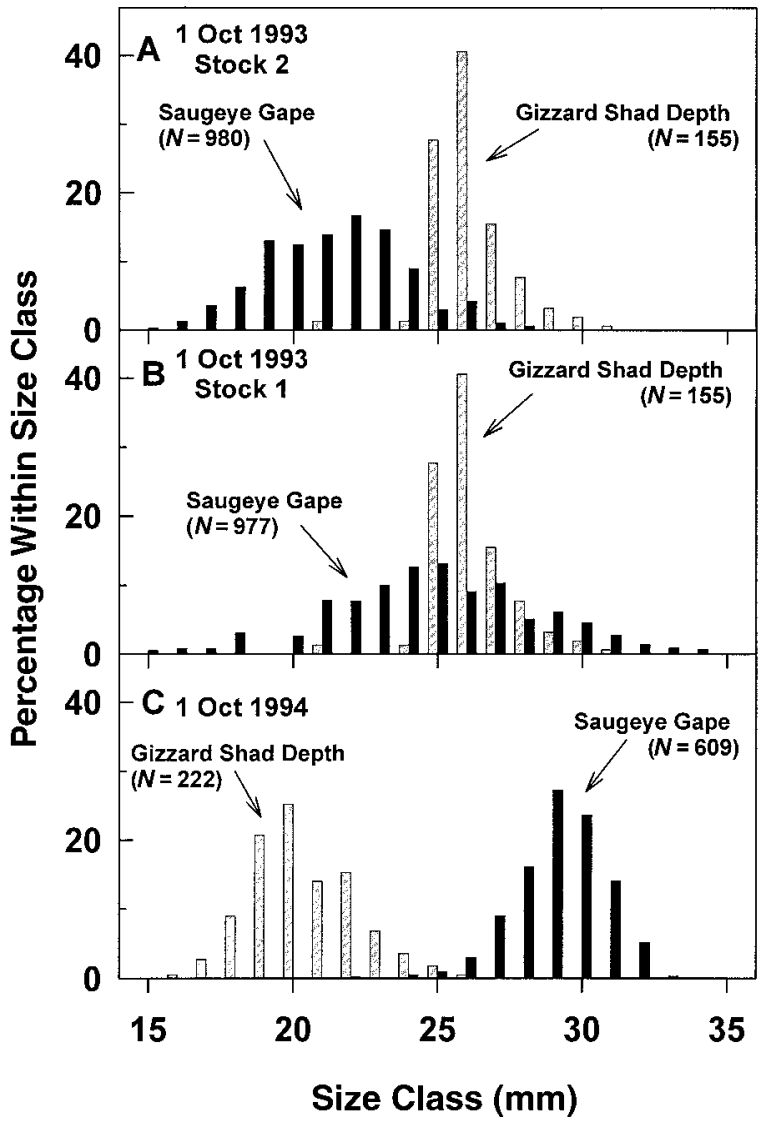

FIG. 6. Comparisons of the percentage of saugeye gapes and gizzard shad depths in 1-mm size classes on 1 October 1993 and 1994, indicating gizzard shad availability to saugeye predators in Pleasant Hill Reservoir, Ohio. (A) In 1993, stock-2 saugeye could consume a smaller proportion of the gizzard shad size distribution than stock-1 saugeye (B). (C) In 1994, saugeye could consume all sizes of gizzard shad. Conversions from total length are described in the Materials and methods: 1994 Reservoir experiment: Analysis of diets. Number included in each distribution is provided in parentheses. Number of saugeye examined for protein type was as in Fig. 5.

ably did not starve. In enclosure and pond experiments, densities of preferred zooplankton mimicked those in Ohio reservoirs during 1991-1994. Thus, high saugeye survival in these experiments (13 and $30 \mathrm{~d}$ ) coupled with no effect of reservoir zooplankton density on saugeye survival suggests that saugeye did not starve. For pond-reared walleyes $(20-50 \mathrm{~mm})$, growth is related to stomach fullness, but not survival (Fox 1989). With flexible foraging behavior, fingerling percids can consume zooplankton or macrobenthos in ponds (Qin et al. 1994 for walleye; pond experiment, this study), become piscivorous (Mathias and Li 1982, Stahl and Stein 1994, this study), or revert to zooplanktivory following stocking (Stahl et al. 1996, this study). Hence, survival of saugeye immediately poststocking was not regulated by zooplankton or piscine prey.

Although fingerling saugeye do not starve, their ov- 


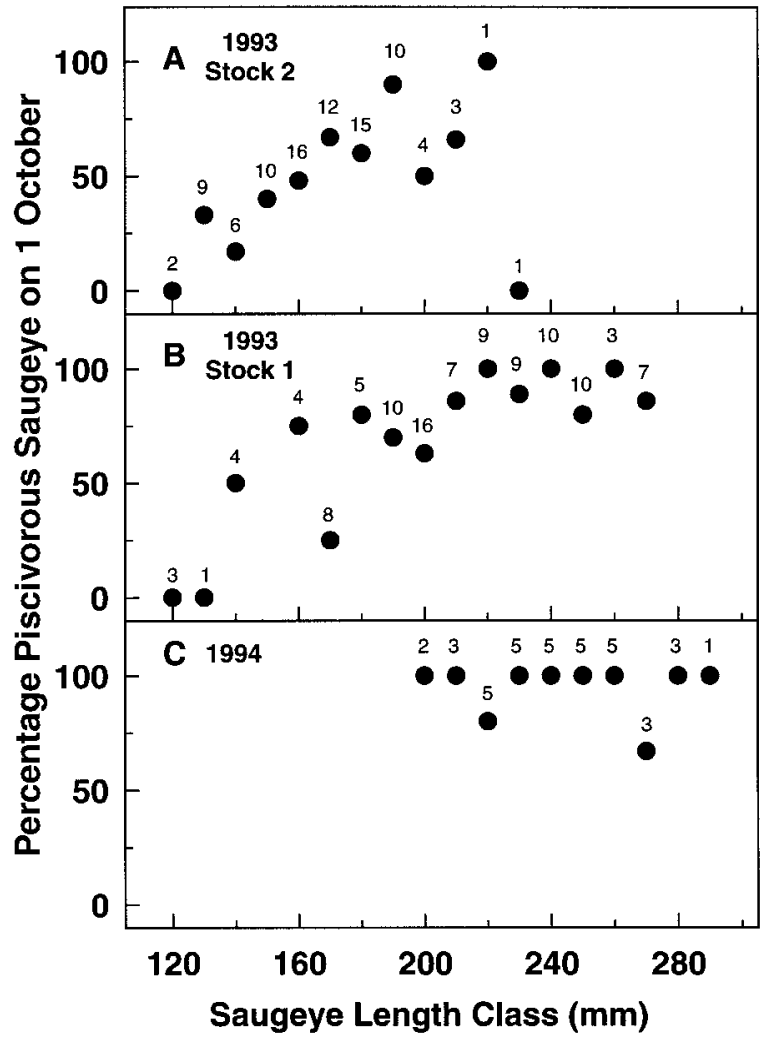

FIG. 7. Percentage of young-of-year saugeye in each 10-mm size class with fish in their diet on 1 October 1993 and 1994 in Pleasant Hill Reservoir, Ohio. In 1993, saugeye length classes, from both stock 2 (A) and stock 1 (B), represented a similar percentage with fish prey; $\geq 75 \%$ of saugeye $>202 \mathrm{~mm}$ had fish prey in their diet. (C) In 1994, virtually all saugeye contained fish prey, as a result of their large size and small gizzard shad size.

ersummer survival still was related to peak gizzard shad density. In 1991 and 1993, when larval gizzard shad peaks occurred early, coinciding with stocking, saugeye survival varied according to peak density. In 1992 and 1994, when larval gizzard shad peaks were delayed, saugeye were stocked in the virtual absence of piscine prey, and their survival was uniformly low $(<6 \%)$, despite eventual peak densities as great as 20 larval gizzard $\mathrm{shad} / \mathrm{m}^{3}$. Given the delay to piscivory by saugeye in all years (see Stahl et al. 1996 for 1991 data), rapid growth coupled with an increasing ability to avoid predation probably does not explain why gizzard shad density positively influenced saugeye survival. Rather, larval gizzard shad probably act to buffer predation on stocked saugeye by resident piscivores such as largemouth bass, smallmouth bass, and age $1+$ saugeye.

Saugeye losses to predators may be size independent as (1) size at stocking and survival to fall were unrelated for all years, (2) most age $1+$ predators can consume stocked saugeye, and (3) most all mortality occurs in the 2 mo poststocking, as suggested by the

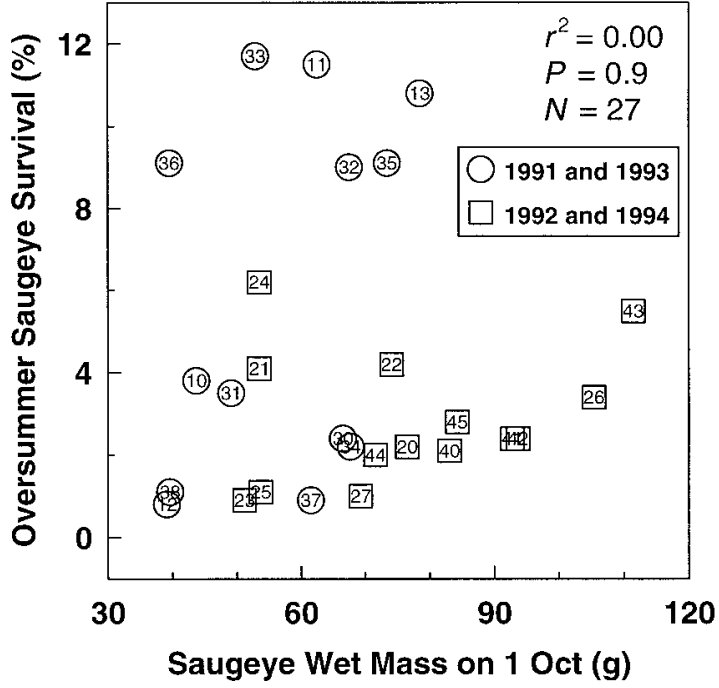

FIG. 8. Percentage oversummer saugeye survival as a function of mean wet mass on 1 October for saugeye stocked into Ohio reservoirs during 1991-1994. For reservoir codes, see Table 1.

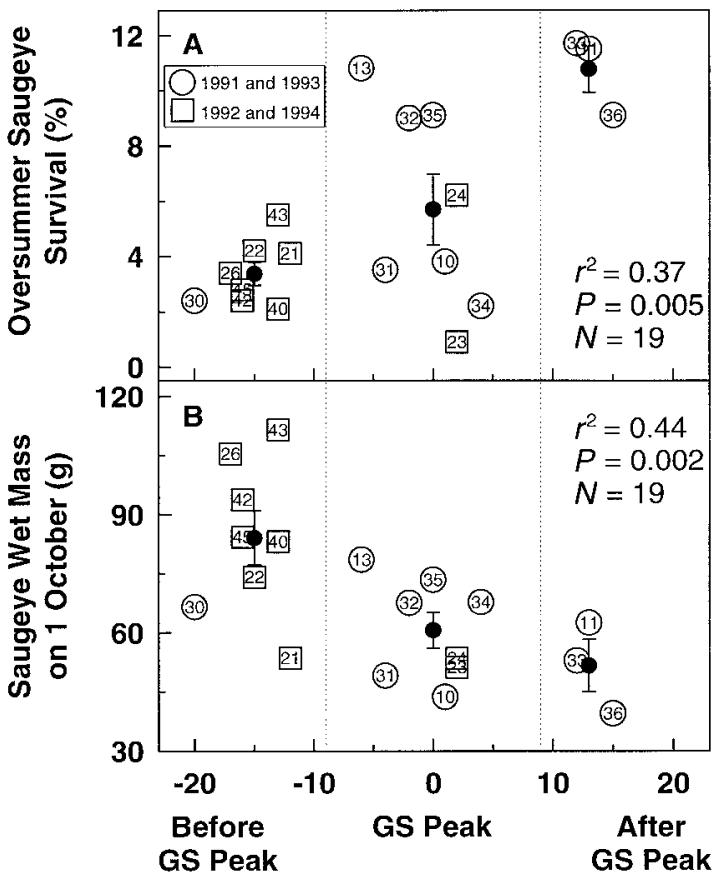

FIG. 9. Oversummer saugeye survival (A) and mean wet mass on 1 October (B) as a function of the relative time of saugeye stocking and the peak density of larval gizzard shad (GS) in Ohio reservoirs during 1991-1994. Negative values on the $x$ axis represent the number of days saugeye were stocked before peak larval gizzard shad density; positive values represent stocking after peak density. Mean ( $\pm 1 \mathrm{SE})$ saugeye survival and wet mass on 1 October are presented for reservoirs stocked early $(<-7)$, average $(-7$ to 7$)$, and late $(>7)$, relative to peak density. Only reservoirs with peaks $\geq 5$ larval gizzard shad $/ \mathrm{m}^{3}$ are presented. 
summer population estimate in Pleasant Hill Reservoir in 1993 (also see Stein et al. 1981, Carline et al. 1986, Stahl et al. 1996). Predation by largemouth bass on $130-160 \mathrm{~mm}$ and $190-220 \mathrm{~mm}$ walleyes is minimal (14 and $0 \%$, respectively) during the months following stocking (Wahl 1995), supporting our conclusion that loss of saugeye to predators is negligible beyond 5 August in Pleasant Hill Reservoir (mean length of stock 1 and stock 2 on 5 August was 146 and $133.9 \mathrm{~mm}$, respectively). As the primary summer forage for most piscivores in Ohio reservoirs (B. M. Johnson et al 1988), YOY gizzard shad cause the predatory focus to shift from stocked saugeye (as saugeye grow sufficiently large to evade capture) to gizzard shad as gizzard shad grow to sizes ( $>25 \mathrm{~mm}$, TL) preferred by resident predators. Finally, using an individual-based model to describe walleye growth during summer, Madenjian and Carpenter (1991) successfully simulated their fall length distributions without incorporating size-selective predation. As such, we propose that larval gizzard shad density positively influences saugeye oversummer survival by buffering predatory pressure on saugeye, as observed in Oneida Lake, where abundant yellow perch (Perca flavescens) buffer walleye cannibalism (Forney 1974).

\section{Prey density and saugeye growth: delayed prey exploitation}

Across three spatial scales (i.e., enclosures, ponds, and reservoirs), saugeye conversion to piscivory was unrelated to larval gizzard shad density. Because saugeye can become piscivorous within $12 \mathrm{~h}$, even at densities of 5 larval gizzard $\mathrm{shad} / \mathrm{m}^{3}$, we could not assess a threshold density below which saugeye do not switch. In ponds, although gizzard shad only occurred in two saugeye $(N=54)$, we believe saugeye did consume gizzard shad, given that peak density was $>4$ times greater in ponds with no saugeye, and gizzard shad were smaller in ponds with saugeye. Using a walleye bioenergetics model (Madon and Culver 1993), Stahl and Stein (1994) demonstrate how, despite being unable to document gizzard shad in saugeye diets, saugeye consumed all larval gizzard shad (71.2 larval gizzard $\mathrm{shad} / \mathrm{m}^{3}$ ) in a hatchery pond within $24 \mathrm{~h}$. Finally, in reservoirs, saugeye conversion to piscivory was delayed 1-5 wk, hypothesized to result from sampling bias, low spatial overlap with gizzard shad, or a preference for small, easily digested prey (Stahl and Stein 1994). As a result, saugeye conversion to fish prey was unrelated to either density or timing of gizzard shad peaks.

Contrary to our hypothesis, saugeye growth was unrelated to larval gizzard shad density. In enclosures with 20 or 100 larval gizzard $\mathrm{shad} / \mathrm{m}^{3}$, but no prey replacement, saugeye grow faster with more gizzard shad (Stahl and Stein 1994). In our enclosures, with prey replacement, saugeye grew faster with larval gizzard shad than without, but similarly across densities of 5, 10, and 20 larval gizzard $\mathrm{shad} / \mathrm{m}^{3}$. Despite our belief that saugeye consumed gizzard shad, saugeye growth did not differ between ponds with and without gizzard shad prey, probably because gizzard shad selected by saugeye were of insufficient mass to allow documentation by diet analysis. Further, in reservoirs, saugeye mass on 1 October was both unrelated to peak gizzard shad density and did not differ among pointand scatter-stocked reservoirs. Even if saugeye did consume gizzard shad immediately following stocking, the lack of a growth response among enclosure treatments suggests that saugeye growth would not be related to larval gizzard shad density in reservoirs. Though larval gizzard shad density immediately following stocking was critical to saugeye survival, it did not immediately influence saugeye growth.

\section{Prey timing and saugeye growth: size-dependent mechanisms}

Rather than influencing saugeye growth directly, peak larval gizzard shad timing foretells the intensity of size-based interactions between saugeye and gizzard shad that would occur later in summer. Because piscivores are gape-limited predators (Hambright et al. 1991) and gizzard shad grow quickly (especially in depth), their vulnerability to predation probably limits oversummer growth of piscivores (largemouth bass, Adams and DeAngelis 1987; walleye and saugeye, B. L. Johnson et al. 1988, B. M. Johnson et al. 1988). The relative impact of gape limitation is mediated by the relative timing of saugeye stocking and gizzard shad spawning. By adjusting early length distributions of shad prey and largemouth bass by just $10 \%$, Adams and DeAngelis (1987) sharply modified fall size distributions (i.e., growth) of largemouth bass. Largemouth bass grew large in years of early largemouth bass and/or delayed shad spawning, but were small in years of late largemouth bass and/or early shad spawning. Similarly, Madenjian et al. (1991) determined that the number of walleye $>175 \mathrm{~mm}$ (a size critical to overwinter survival) in fall could be increased by $\sim 3$ times by either stocking walleye of a given size $2 \mathrm{wk}$ earlier, or increasing size at stocking by $10 \mathrm{~mm}$. Our empirical saugeye growth data reinforce these modeling results, increasing their generality and robustness.

For 1993 and 1994, saugeye were largest in 1994, followed by stock 1 of 1993 , then stock 2, likely in response to late gizzard shad production in 1994. In Pleasant Hill Reservoir in 1994, almost all saugeye contained fish, allowing continued rapid growth through 1 October. In 1993, both stocks grew at similar rates through July. By 5 August, these two stocks had begun to diverge in size, with stock 1 being $12 \mathrm{~mm}$ larger, on average, than stock 2. Because larger stock-1 fish had more access to rapidly growing gizzard shad than those of stock 2, stock 1 grew faster through early fall and were $30.8 \mathrm{~mm}(33.9 \mathrm{~g})$ larger than stock 2 by 1 October. This example emphasizes how small dif- 
ferences in size can lead to large differences in available prey, growth rate, and end-of-season size. These empirical results support modeling conclusions (Adams and DeAngelis 1987, Madenjian and Carpenter 1991) that a small size advantage, early in the growing season, can compound through time. Thus, individuals at the leading edge of a size distribution early in the season ultimately enjoy substantially improved growth and survival.

\section{Overwinter survival}

For some fishes, overwinter can be the final critical period before recruiting as adults (walleye, Forney 1976; Esox spp., Carline et al. 1986; yellow perch, Post and Evans 1989; largemouth bass, Ludsin and DeVries 1997). Though Forney (1976) reported increased survival of walleye $>175 \mathrm{~mm}$ entering the winter, we could document no size-selective overwinter mortality for saugeye as small as $113 \mathrm{~mm}$ survived to spring. Severity of size-selective overwinter mortality may vary among species and along a latitudinal gradient, reflecting varying ability to regulate metabolic activity during thermal stress and low food availability (Sullivan 1986, Shuter and Post 1990). For percids, which continue to feed through winter, high activity rate during long cold winters may compromise overwinter survival in northern systems (Forney 1976, Post and Evans 1989). In southern systems (perhaps including Ohio reservoirs), reduced thermal stress and shorter winters combine to allow greater overwinter survival (Sullivan 1986).

\section{Management recommendations}

Through a partnership with Ohio Division of Wildlife (sensu Parrish et al. 1995), with the common goal of improving saugeye angling opportunities in Ohio, we have employed adaptive management techniques (sensu Walters 1986) to further illuminate the mechanisms that regulate saugeye recruitment. Only through the marriage of multiscale experiments and multiple years of whole-reservoir experiments testing explicit hypotheses were we able to discard our original hypothesis that early use of gizzard shad prey increases saugeye growth and survival oversummer. By coupling our experiments, which demonstrate how timing of saugeye stocking and gizzard shad spawning influence saugeye growth and survival, with individual-based modeling efforts (Adams and DeAngelis 1987, Madenjian and Carpenter 1991, Madenjian et al. 1991), we can propose management strategies to increase stocking success. Fisheries managers can sample reservoirs for the critically variable element (in our case, timing of gizzard shad hatching) and stock according to their management goals of either greater growth or survival of young-of-year saugeye. In this context, a model that might predict when gizzard shad peaks occur would be quite valuable. Even without this model, managers can follow the guideline that $\sim 70 \%$ of gizzard shad peaks in Ohio reservoirs occur during 22 May-11 June ( $N=$ 62 reservoir-years; M. T. Bremigan, Aquatic Ecology Laboratory, The Ohio State University, unpublished data). Managers also must recognize that later stocking for survival may delay recruitment of saugeye to the fishery. Given that early-stocked saugeye often recruit to the fishery their first fall, fewer fish might ultimately be harvested if overwinter mortality is substantial. Because gizzard shad density (perhaps expressed as number of prey per predator) early in life does not influence saugeye growth, then "scatter" stocking makes little sense. Rather, if our earlier conjecture is correct, predation probably drives early survival of saugeye and "point" stocking would serve to swamp predator populations, providing the best opportunity for survival immediately poststocking. Conceivably, some reservoirs may support low densities of predators; in these systems, managers may be able to stock early, gaining both growth and survival advantages. In more typical predator-abundant systems with gizzard shad as prey, reservoir managers should point stock saugeye (1) early for best growth or (2) later for best survival. By manipulating stock date relative to larval gizzard shad peaks, Ohio fisheries managers probably either can increase saugeye size or survival to fall, but not both.

\section{ACKNOWLEDGMENTS}

We are grateful for the efforts of the many individuals that contributed technical and theoretical assistance to this research. In particular, Michele Donovan, Lesley Fisher, and Gayle Martin provided exemplary assistance throughout this study. Ohio Division of Wildlife (ODW) personnel were instrumental to this research: Milt Austin, Gary Isbell, and Randy Miller provided leadership critical to the cooperation between ODW and OSU; Pat Keyes and Jim Stafford provided technical expertise and hatchery facilities; and individuals from all five Ohio districts provided valuable system insight and assistance in sample collection. Sadly, Milt Austin, coordinator of the ODW saugeye effort, died suddenly just prior to the completion of this work. His professionalism and commitment to project goals and aquatic resources were a model for us all. Chuck Madenjian and Libby Marschall provided constructive critical reviews of an earlier draft. Tom Stahl set an impressive standard through his early work with saugeye at the Aquatic Ecology Laboratory. We also thank the faculty, graduate students, and technicians of the Aquatic Ecology Laboratory for helpful comments and assistance. This work was funded by the Federal Aid in Sport Fish Restoration Program (F-69-P, Evaluation of Fish Management Techniques), administered jointly by the U.S. Fish and Wildlife Service and the Ohio Division of Wildlife.

\section{Literature Cited}

Adams, S. M., and D. L. DeAngelis. 1987. Indirect effects of early bass-shad interactions on predator population structure and food web dynamics. Pages 103-117 in W. C. Kerfoot and A. Sih, editors. Predation: direct and indirect impact on aquatic communities. University Press of New England, Hanover, New Hampshire, USA.

Adams, S. M., R. B. McLean, and M. M. Huffman. 1982. Structuring of a predator population through temperaturemediated effects on prey availability. Canadian Journal of Fisheries and Aquatic Sciences 39:1175-1184.

Aggus, L. R., and G. V. Elliott. 1975. Effects of cover and food on year-class strength of largemouth bass. Pages 317- 
322 in R. H. Stroud, editor. Black bass biology and management. Sport Fishing Institute, Washington, D.C., USA.

Antonovics, J., and D. A. Levin. 1980. The ecological and genetic consequences of density-dependent regulation in plants. Annual Review of Ecology and Systematics 11:411452 .

Baylis, J. R., D. D. Wiegmann, and M. H. Hoff. 1993. Alternating life histories of smallmouth bass. Transactions of the American Fisheries Society 122:500-510.

Buijse, A. D., and R. P. Houthuijzen. 1992. Piscivory, growth, and size-selective mortality of age 0 pikeperch (Stizostedion lucioperca). Canadian Journal of Fisheries and Aquatic Sciences 49:894-902.

Carline, R. F., R. A. Stein, and L. W. Riley. 1986. Effects of size at stocking, season, largemouth bass predation, and forage abundance on survival of tiger muskellunge. American Fisheries Society Special Publication 15:135-150.

Condit, R., S. P. Hubbell, and R. B. Foster. 1994. Density dependence in two understory tree species in a neotropical forest. Ecology 75:671-680.

Connell, J. H., J. G. Tracey, and L. J. Webb. 1984. Compensatory recruitment, growth, and mortality as factors maintaining rain forest tree diversity. Ecological Monographs 54:141-164.

Crecco, V. A., and T. F. Savoy. 1985. Effects of biotic and abiotic factors on growth and relative survival of young American shad, Alosa sapidissima, in the Connecticut River. Canadian Journal of Fisheries and Aquatic Sciences 42: 1640-1648.

Crowder, L. B. 1980. Alewife, rainbow smelt and native fishes in Lake Michigan: competition or predation? Environmental Biology of Fishes 5:225-233.

Culver, D. A., S. P. Madon, and J. Qin. 1993. Percid pond production techniques: timing, enrichment, and stocking density manipulation. Journal of Applied Aquaculture 2 9-31.

Cushing, D. H. 1975. Marine ecology and fisheries. Cambridge University Press, Cambridge, UK.

DeAngelis, D. L., and C. C. Coutant. 1982. Genesis of bimodal size distributions in species cohorts. Transactions of the American Fisheries Society 111:384-388.

Dettmers, J. M., and R. A. Stein. 1992. Food consumption by larval gizzard shad: zooplankton effects and implications for reservoir communities. Transactions of the American Fisheries Society 121:494-507.

DeVries, D. R., and R. A. Stein. 1991. Comparison of three zooplankton samplers: a taxon-specific assessment. Journal of Plankton Research 13:53-59.

Doherty, P., and T. Fowler. 1994. An empirical test of recruitment limitation in a coral reef fish. Science 263:935939.

Donovan, N. S., T. P. Stahl, R. A. Stein, M. R. Fore, L. C. Fisher, and M. T. Bremigan. 1996. Evaluating saugeye stocking success in Ohio reservoirs. Final Report, Federal Aid in Sport Fish Restoration Project F-69-P Study FADR 20, Ohio Department of Natural Resources, Division of Wildlife, Columbus, Ohio, USA.

Dumont, H. J., I. Van De Velde, and S. Dumont. 1975. The dry weight estimate of biomass in a selection of Cladocera, Copepoda and Rotifera from the plankton, periphyton, and benthos of continental waters. Oecologia 19:75-97.

Fielder, D. G. 1992. Evaluation of stocking walleye fry and fingerlings and factors affecting their success in Lower Lake Oahe, South Dakota. North American Journal of Fisheries Management 12:336-345.

Forney, J. L. 1974. Interactions between yellow perch abundance, walleye predation, and survival of alternate prey in Oneida Lake, New York. Transactions of the American Fisheries Society 103:15-24.

. 1976. Year-class formation in the walleye (Stizoste- dion vitreum vitreum) population of Oneida Lake, New York, 1966-73. Journal of the Fisheries Research Board of Canada 33:783-792.

Fox, M. G. 1989. Effect of prey density and prey size on growth and survival of juvenile walleye (Stizostedion vitreum vitreum). Canadian Journal of Fisheries and Aquatic Sciences 46: $1323-1328$.

Gutreuter, S. J., and R. O. Anderson. 1985. Importance of body size to the recruitment process in largemouth bass populations. Transactions of the American Fisheries Society 114:317-327.

Hambright, K. D., R. W. Drenner, S. J. McComas, and N. G. Hairston, Jr. 1991. Gape-limited piscivores, planktivore size refuges, and the trophic cascade hypothesis. Hydrobiologia 121:389-404

Healey, M. C. 1982. Timing and relative intensity of sizeselective mortality of juvenile chum salmon (Oncorhynchus keta) during early sea life. Canadian Journal of Fisheries and Aquatic Sciences 39:952-957.

Houde, E. D. 1987. Fish early life dynamics and recruitment variability. American Fisheries Society Symposium 2:1729.

1994. Differences between marine and freshwater fish larvae: implications for recruitment. Journal du Conseil International pour l'Exploration de la Mer 51:91-97.

Hurley, S. T., and M. R. Austin. 1987. Evaluation of walleye stocking in Caesar Creek Lake. Ohio Department of Natural Resources, Federal Aid in Fish Restoration, Project F-29-R, Completion Report, Columbus, Ohio, USA.

Jackson, J. J., D. W. Willis, and D. G. Fielder. 1992. Food habits of young-of-the-year walleyes in Okobojo Bay of Lake Oahe, South Dakota. Journal of Freshwater Ecology 7:329-341.

Jernejcic, F. 1986. Walleye migration through Tygart Dam and angler utilization of the resulting tailwater and lake fisheries. Pages 294-300 in G. E. Hall and M. J. Van Den Avyle, editors. Reservoir fisheries management: strategies for the 80's. Reservoir Committee, Southern Division American Fisheries Society, Bethesda, Maryland, USA.

Johnson, B. L. 1981. First-year growth, survival, habitat preference, and food habits of stocked walleye and walleye × sauger hybrids in Pleasant Hill Reservoir, Ohio. Master's thesis. The Ohio State University, Columbus, Ohio, USA.

Johnson, B. L., D. L. Smith, and R. F. Carline. 1988. Habitat preferences, survival, growth, foods, and harvest of walleyes and walleye $\times$ sauger hybrids. North American Journal of Fisheries Management 8:292-304.

Johnson, B. M., R. A. Stein, and R. F. Carline. 1988. Use of a quadrat rotenone technique and bioenergetics modeling to evaluate prey availability to stocked piscivores. Transactions of the American Fisheries Society 117:127-141.

Kramer, R. H., and L. L. Smith, Jr. 1962. Formation of the year-classes in largemouth bass. Transactions of the American Fisheries Society 91:29-41.

Laarman, P. W. 1978. Case histories of stocking walleyes in inland lakes, impoundments, and the Great Lakes-100 years with walleyes. American Fisheries Society Special Publication 11:254-260.

Lasker, R. 1975. Field criteria for survival of anchovy larvae: the relation between inshore chlorophyll maximum layers and successful first feeding. Fisheries Bulletin 73:453-462.

Lauenroth, W. K., O. E. Sala, D. P. Coffin, and T. B. Kirchner. 1994. The importance of soil water in the recruitment of Bouteloua gracilis in the shortgrass steppe. Ecological Applications 4:741-749.

Lawrence, J. M. 1960. Estimated sizes of various forage fishes chain pickerel can swallow. Proceedings of the Southeastern Association of Game and Fish Commission 14:257-258.

Li, S., and J. A. Mathias. 1982. Causes of high mortality 
among cultured larval walleye. Transactions of the American Fisheries Society 111:710-721.

Louda, S. M., M. A. Potvin, and S. K. Collinge. 1990. Predispersal seed predation, postdispersal seed predation and competition in the recruitment of seedlings of a native thistle in Sandhills prairie. American Midland Naturalist 124: $105-113$.

Ludsin, S. A., and D. R. DeVries. 1997. First-year recruitment of largemouth bass: the inter-dependency of early life stages. Ecological Applications 7(3):000-000.

Luecke, C., J. A. Rice, L. B. Crowder, S. E. Yeo, and F. P Binkowski. 1990. Recruitment mechanisms of bloater in Lake Michigan: an analysis of the predatory gauntlet. Canadian Journal of Fisheries and Aquatic Sciences 47:524532

Madenjian, C. P., and S. R. Carpenter. 1991. Individual-based model for growth of young-of-the-year walleye: a piece of the recruitment puzzle. Ecological Applications 1:268-279.

Madenjian, C. P., B. M. Johnson, and S. R. Carpenter. 1991 Stocking strategies for fingerling walleyes: an individualbased model approach. Ecological Applications 1:280288.

Madon, S. P., and D. A. Culver. 1993. Bioenergetics model for larval and juvenile walleye: an in-situ approach using experimental ponds. Transactions of the American Fisheries Society 122:797-813.

Malison, J. A., T. B. Kayes, J. A. Held, and C. H. Amundson. 1990. Comparative survival, growth, and reproductive development of juvenile walleye and sauger and their hybrids reared under intensive culture conditions. Progressive FishCulturist 52:73-82.

Marschall, E. A., and L. B. Crowder. 1995. Density-dependent survival as a function of size in juvenile salmonids in streams. Canadian Journal of Fisheries and Aquatic Sciences 52:136-140.

Mathias J. A., and S. Li. 1982. Feeding habits of walleye larvae and juveniles: comparative laboratory and field studies. Transactions of the American Fisheries Society 111 722-735

May, R. C. 1974. Larval mortality in marine fishes and the critical period concept. Pages 3-19 in J. H. S. Blaxter editor. The early life history of fish. Springer-Verlag, New York, New York, USA.

Miller, T. J., L. B. Crowder, J. A. Rice, and E. A. Marschall. 1988. Larval size and recruitment mechanisms in fishes: toward a conceptual framework. Canadian Journal of Fisheries and Aquatic Sciences 45:1657-1670.

Mittelbach, G. G. 1981. Foraging efficiency and body size: a study of optimal diet and habitat use by bluegills. Ecology 62:1370-1386.

Morizot, D. C., and M. E. Schmidt. 1990. Starch gel electrophoresis and histochemical visualization of proteins. Pages 23-80 in D. H. Whitmore, editor. Electrophoretic and isoelectric focusing techniques in fisheries management. CRC Press, Boca Raton, Florida, USA.

Oliver, J. D., G. F. Holeton, and K. E. Chus. 1979. Overwinter mortality of fingerling smallmouth bass in relation to size, relative energy stores, and environmental temperature. Transactions of the American Fisheries Society 108: 130-136.

Olson, M. H. 1996. Ontogenetic niche shifts in largemouth bass: variability and consequences for first-year growth. Ecology 77:179-190.

Paine, R. T. 1976. Size-limited predation: an observational and experimental approach with the Mytilus-Pisaster interaction. Ecology 57:858-873.

Parrish, D. L., M. E. Mather, and R. A. Stein. 1995. Problemsolving research for management: a perspective. Fisheries 20(10):6-12.

Phillips, J. M., J. R. Jackson, and R. L. Noble. 1995. Hatch- ing date influence on age-specific diet and growth of age-0 largemouth bass. Transactions of the American Fisheries Society 124:370-379.

Post, J. R., and D. O. Evans. 1989. Size-dependent overwinter mortality of young-of-the-year yellow perch (Perca flavescens): laboratory, in situ enclosure, and field experiments. Canadian Journal of Fisheries and Aquatic Sciences 46: $1958-1968$

Post, J. R., and A. B. Prankevicius. 1987. Size-selective mortality in young-of-the-year yellow perch (Perca flavescens): evidence from otolith microstructure. Canadian Journal of Fisheries and Aquatic Sciences 44:1840-1847.

Qin, J., D. A. Culver, and N. Yu. 1994. Comparisons of larval walleye and saugeye (walleye $\times$ sauger hybrid) growth and impacts on zooplankton in experimental ponds. Progressive Fish-Culturist 56:91-99.

Rice, J. A., L. B. Crowder, and F. P. Binkowski. 1987a. Evaluating potential sources of mortality for larval bloater (Coregonus hoyi): starvation and vulnerability to predation. Canadian Journal of Fisheries and Aquatic Sciences 44:467472.

Rice, J. A., L. B. Crowder, and M. E. Holey. 1987b. Exploration of mechanisms regulating larval survival in Lake Michigan bloater: a recruitment analysis based on characteristics of individual larvae. Transactions of the American Fisheries Society 116:703-718.

Rice, J. A., T. J. Miller, K. A. Rose, L. B. Crowder, E. A. Marschall, A. S. Trebitz, and D. L. DeAngelis. 1993. Growth rate variation and larval survival: inferences from an individual-based size-dependent predation model. Canadian Journal of Fisheries and Aquatic Sciences 50:133142.

Santucci, V. J., Jr., and D. H. Wahl. 1993. Factors influencing survival and growth of stocked walleye (Stizostedion vitreum) in a centrarchid-dominated impoundment. Canadian Journal of Fisheries and Aquatic Sciences 50:1548-1558.

Shuter, B. J., and J. R. Post. 1990. Climate, population viability, and the zoogeography of temperate fishes. Transactions of the American Fisheries Society 119:314-336.

Siegwarth, G. L., and R. C. Summerfelt. 1990. Growth comparisons between fingerling walleyes and walleye $\times$ sauger hybrids reared in intensive culture. Progressive Fish-Culturist 52:100-104.

Stahl, T., N. S. Donovan, R. A. Stein, and D. A. Culver. 1993. Evaluating saugeye stocking success in Ohio reservoirs. Annual Report, Federal Aid in Sport Fish Restoration Project F-57-R Study 20, Ohio Department of Natural Resources, Division of Wildlife, Columbus, Ohio, USA

Stahl, T., and R. A. Stein. 1994. The influence of larval gizzard shad density on piscivory and growth of youngof-year saugeye (Stizostedion vitreum vitreum $\times S$. canadense). Canadian Journal of Fisheries and Aquatic Sciences 51:1993-2002.

Stahl, T. P., G. P. Theide, R. A. Stein, E. M. Lewis, M. R. Austin, and D. A. Culver. 1996. Factors affecting survival of young-of-year saugeye (Stizostedion vitreum vitreum $\times$ S. canadense) stocked in Ohio reservoirs. North American Journal of Fisheries Management 16:378-387.

Stein, R. A., R. F. Carline, and R. S. Hayward. 1981. Largemouth bass predation on stocked tiger muskellunge. Transactions of the American Fisheries Society 110:604-612.

Stein, R. A., P. E. Reimers, and J. D. Hall. 1972. Social interaction between juvenile coho (Oncorhynchus kisutch) and fall chinook salmon (O. tshawytscha) in Sixes River, Oregon. Journal of the Fisheries Research Board of Canada 29:1737-1748.

Sullivan, K. M. 1986. Physiology of feeding and starvation tolerance in overwintering freshwater fishes. Developmental and Environmental Biology of Fishes 7:259-268.

Thompson, J. M., E. P. Bergersen, C. A. Carlson, and L. R. 
Kaeding. 1991. Role of size, condition, and lipid content in the overwinter survival of age-0 Colorado squawfish. Transactions of the American Fisheries Society 120:346353

Todd, T. N. 1990. Genetic differentiation of walleye stocks in Lake St. Clair and western Lake Erie. U.S. Fish and Wildlife Service Technical Report 28.

Turner, B. J. 1983. Genetic variation and differentiation of remnant natural populations of the desert pupfish, Cyprinodon macularius. Evolution 37:690-700.

Uthe, J. F., and R. A. Ryder. 1970. Regional variation in muscle myogen polymorphism in walleye (Stizostedion vitreum vitreum) as related to morphology. Journal of the Fisheries Research Board of Canada 27:923-927.

Victor, B. C. 1986. Larval settlement and juvenile mortality in a recruitment-limited coral reef fish population. Ecological Monographs 56:145-160.
Wahl, D. H. 1988. Comparison of population ecology in two species of esocids and their hybrid. Dissertation. The Ohio State University, Columbus, Ohio, USA.

- 1995. Effect of habitat selection and behavior on vulnerability to predation of introduced fish. Canadian Journal of Fisheries and Aquatic Sciences 52:2312-2319.

Wahl, D. H., and R. A. Stein. 1989. Comparative vulnerability of three esocids to largemouth bass (Micropterus salmoides) predation. Canadian Journal of Fisheries and Aquatic Sciences 46:2095-2103.

Walters, C. J. 1986. Adaptive management of renewable resources. Macmillan, New York, New York, USA.

Werner, E. E., and J. F. Gilliam. 1984. The ontogenetic niche and species interactions in size-structured populations. Annual Review of Ecology and Systematics 15:393-425.

White, M. M., and S. Schell. 1995. An evaluation of the genetic integrity of Ohio River walleye and sauger stocks. American Fisheries Society Symposium 15:52-60. 